DiRECTEUR DE LA PUBLICATION: Bruno David,

Président du Muséum national d'Histoire naturelle

RÉdACTEUR EN CHEF / EDITOR-IN-CHIEF: Didier Merle

ASSISTANTS DE RÉDACTION / AsSISTANT EDITORS: Emmanuel Côtez (geodiv@mnhn.fr); Anne Mabille

Mise EN PAGE / PAGE LAYOUT: Emmanuel Côtez

COMITÉ SCIENTIFIQUE / SCIENTIFIC BOARD:

Christine Argot (MNHN, Paris)

Beatrix Azanza (Museo Nacional de Ciencias Naturales, Madrid)

Raymond L. Bernor (Howard University, Washington DC)

Alain Blieck (chercheur CNRS retraité, Haubourdin)

Henning Blom (Uppsala University)

Jean Broutin (UPMC, Paris)

Gaël Clément (MNHN, Paris)

Ted Daeschler (Academy of Natural Sciences, Philadelphie)

Bruno David (MNHN, Paris)

Gregory D. Edgecombe (The Natural History Museum, Londres)

Ursula Göhlich (Natural History Museum Vienna)

Jin Meng (American Museum of Natural History, New York)

Brigitte Meyer-Berthaud (CIRAD, Montpellier)

Zhu Min (Chinese Academy of Sciences, Pékin)

Isabelle Rouget (UPMC, Paris)

Sevket Sen (MNHN, Paris)

Stanislav Štamberg (Museum of Eastern Bohemia, Hradec Králové)

Paul Taylor (The Natural History Museum, Londres)

COUVERTURE / COVER:

Réalisée à partir des figures de l'article/Made from the Figures of the article.

Geodiversitas est indexé dans / Geodiversitas is indexed in:

- Science Citation Index Expanded (SciSearch ${ }^{\circledR}$ )

- ISI Alerting Services ${ }^{\circledR}$

- Current Contents ${ }^{\circledR}$ / Physical, Chemical, and Earth Sciences ${ }^{\circledR}$

- Scopus ${ }^{\circledR}$

Geodiversitas est distribué en version électronique par / Geodiversitas is distributed electronically by:

- BioOne ${ }^{\circledR}$ (http://www.bioone.org)

Les articles ainsi que les nouveautés nomenclaturales publiés dans Geodiversitas sont référencés par / Articles and nomenclatural novelties published in Geodiversitas are referenced by:

- ZooBank ${ }^{\circledR}$ (http://zoobank.org)

Geodiversitas est une revue en flux continu publiée par les Publications scientifiques du Muséum, Paris Geodiversitas is a fast track journal published by the Museum Science Press, Paris

Les Publications scientifiques du Muséum publient aussi / The Museum Science Press also publish:

Adansonia, Zoosystema, Anthropozoologica, European Journal of Taxonomy, Naturae, Cryptogamie sous-sections Algologie, Bryologie, Mycologie

Diffusion - Publications scientifiques Muséum national d'Histoire naturelle

CP $41-57$ rue Cuvier F-75231 Paris cedex 05 (France)

Tél. : 33 (0)1407948 05 / Fax: 33 (0)14079 3840

diff.pub@mnhn.fr / http://sciencepress.mnhn.fr

(C) Publications scientifiques du Muséum national d'Histoire naturelle, Paris, 2019

ISSN (imprimé / print): 1280-9659/ ISSN (électronique / electronic): 1638-9395 


\title{
Restes inédits de rongeurs caviomorphes du Paléogène de la région de Juanjui (Amazonie péruvienne): systématique, implications macro-évolutives et biostratigraphiques
}

\author{
Alexandre ASSEMAT \\ Myriam BOIVIN \\ Laurent MARIVAUX \\ Laboratoire de Paléontologie, Institut des Sciences de l'Évolution de Montpellier (ISE-M), \\ c.c. 064 , Université de Montpellier, CNRS, IRD, EPHE, \\ place Eugène Bataillon, F-34095 Montpellier cedex 05 (France) \\ alexandre.assemat@gmail.com \\ myriam.boivin@umontpellier.fr \\ laurent.marivaux@umontpellier.fr
}

François PUJOS

Instituto Argentino de Nivología, Glaciología y Ciencias Ambientales (IANIGLA), CCT-CONICET-Mendoza, Av. Ruiz Leal s/n, Parque Gral. San Martín, 5500 Mendoza (Argentina) fpujos@mendoza-conicet.gob.ar

Aldo BENITES-PALOMINO

Departamento de Paleontología de Vertebrados, Museo de Historia Natural - Universidad Nacional Mayor San Marcos (MUSM), Av. Arenales 1256, Lima 11 (Peru) and Center for Tropical Paleoecology and Archeology, Smithsonian Tropical Research Institute, Balboa, Ancon AA 0843-03092 (Panama) aldomar1955@gmail.com

\section{Rodolfo SALAS-GISMONDI}

Departamento de Paleontología de Vertebrados, Museo de Historia Natural - Universidad Nacional Mayor San Marcos (MUSM), Av. Arenales 1256, Lima 11 (Peru) rsalasgismondi@gmail.com

Julia V. TEJADA-LARA Columbia University in the City of New York and Division of Vertebrate Paleontology, American Museum of Natural History, Central Park West at 79th Street, New York, NY 10024 (United States) and Departamento de Paleontología de Vertebrados, Museo de Historia Natural Universidad Nacional Mayor San Marcos (MUSM), Av. Arenales 1256, Lima 11 (Peru) julia.tejada@columbia.edu

Rafael M. VARAS-MALCA Departamento de Paleontología de Vertebrados, Museo de Historia Natural - Universidad Nacional Mayor San Marcos (MUSM), Av. Arenales 1256, Lima 11 (Peru) paleomind@gmail.com

Francisco Ricardo NEGRI

Laboratório de Paleontologia, Campus Floresta, Universidade Federal do Acre, Campus Floresta/Cruzeiro do Sul, Rua Paraná, 860, 69980-000, Cruzeiro do Sul, AC (Brazil) 
Ana Maria RIBEIRO

Seção de Paleontologia, Museu de Ciências Naturais, Fundação Zoobotânica do Rio Grande do Sul, Av. Salvador França 1427, 90690-000, Porto Alegre, RS (Brazil) ana-ribeiro@fzb.rs.gov.br

Pierre-Olivier ANTOINE Laboratoire de Paléontologie, Institut des Sciences de l'Évolution de Montpellier (ISE-M), c.c. 064, Université de Montpellier, CNRS, IRD, EPHE, place Eugène Bataillon, F-34095 Montpellier cedex 05 (France) pierre-olivier.antoine@umontpellier.fr
MOTS CLÉS

Pérou,

Éocène,

Oligocène,

Caviomorpha, morphologie dentaire, biostratigraphie, microstructure de l'émail.

KEY WORDS

Peru,

Eocene,

Oligocene,

Caviomorpha,

biostratigraphy,

enamel microstructure.
Soumis le 14 septembre 2018 | accepté le 15 mai 2019 | publié le 24 octobre 2019

Assemat A., Boivin M., Marivaux L., Pujos F., Benites-Palomino A., Salas-Gismondi R., Tejada-Lara J. V., Varas-Malca R. M., Negri F. R., Ribeiro A. M. \& Antoine P.-O. 2019. - Restes inédits de rongeurs caviomorphes du Paléogène de la région de Juanjui (Amazonie péruvienne): systématique, implications macro-évolutives et biostratigraphiques. Geodiversitas 41 (20): 699-730. https://doi.org/10.5252/geodiversitas2019v41a20. http://geodiversitas.com/41/20

\section{RÉSUMÉ}

Le Paléogène d'Amazonie péruvienne a livré les plus anciennes communautés de rongeurs caviomorphes d'Amérique du Sud, lesquelles sont le résultat des premières phases de diversification de ce groupe. Ce travail présente l'étude de restes dentaires inédits de rongeurs fossiles issus de deux nouvelles sections situées à proximité des villes de Juanjui et de Balsayacu (Département de San Martín, Amazonie péruvienne). Les analyses de la morphologie occlusale des dents jugales et de la microstructure de l'émail des incisives indiquent la présence de taxons basaux tels que les genres Cachiyacuy Antoine, Marivaux, Croft, Billet, Ganerød, Jaramillo, Martin, Orliac, Tejada, Altamirano, Duranthon, Fanjat, Rousse \& Salas Gismondi, 2012, Canaanimys Antoine, Marivaux, Croft, Billet, Ganerød, Jaramillo, Martin, Orliac, Tejada, Altamirano, Duranthon, Fanjat, Rousse \& Salas Gismondi, 2012, et Eoespina Frailey \& Campbell, 2004, dans les niveaux inférieurs de la série sédimentaire, auxquels succèdent des taxons plus dérivés, comme Eoincamys Frailey \& Campbell, 2004, dans des niveaux plus récents. Ces taxons témoignent d'une morphologie intermédiaire entre ceux documentés en Amazonie péruvienne dans les localités éocènes de Contamana (fin de l'Éocène moyen) et ceux des localités oligocènes de Tarapoto/Shapaja et Santa Rosa (?Éocène supérieur/Oligocène inférieur). Ces fossiles apportent ainsi une documentation inédite en Amazonie, en ce que les secteurs de Juanjui et de Balsayacu présentent des dépôts sédimentaires couvrant vraisemblablement la fin de l'Éocène moyen, une partie de l'Éocène supérieur et la base de l'Oligocène. Enfin, la continuité stratigraphique de la longue section de Juanjui permet de démontrer l'existence d'un hiatus temporel significatif entre les assemblages à Canaanimys-Cachiyacuy-Potamotrygon ucayalensis (Contamana, Formation Pozo; base de la section de Juanjui; Balsayacu) et ceux à Eoincamys (Santa Rosa; Tarapoto/ Shapaja; sommet de la section de Juanjui).

\section{ABSTRACT}

New remains of caviomorph rodents from the Paleogene of the Juanjui area (Peruvian Amazonia): systematics, macroevolutionary implications and biostratigraphy.

Paleogene deposits of Peruvian Amazonia have yielded the oldest caviomorph rodent communities from South America, and the clues that their early diversification had occurred in this area. Here we report fossil dental remains of rodents from two new sections located in the vicinity of Juanjui and Balsayacu (San Martín Department, Peruvian Amazonia). Analyses of the occlusal morphology of cheek teeth and of the incisor enamel microstructure indicate the occurrence of basal caviomorphs (such as Cachiyacuy Antoine, Marivaux, Croft, Billet, Ganerød, Jaramillo, Martin, Orliac, Tejada, Altamirano, Duranthon, Fanjat, Rousse \& Salas Gismondi, 2012, Canaanimys Antoine, Marivaux, Croft, Billet, Ganerød, Jaramillo, Martin, Orliac, Tejada, Altamirano, Duranthon, Fanjat, Rousse \& Salas Gismondi, 2012, and Eoespina Frailey \& Campbell, 2004, genera so far known from late middle Eocene deposits of Contamana, Peruvian Amazonia) in levels situated in the lower part of the studied stratigraphic section. More derived taxa (such as Eoincamys Frailey \& Campbell, 2004), known from early Oligocene deposits of Tarapoto/ Shapaja and Santa Rosa, Peruvian Amazonia) occur only in upper levels of the section. Based on these considerations, the rodent-bearing localities of the Juanjui/Balsayacu area seem to be intermediate in age between the Contamana Eocene localities and the Tarapoto/Shapaja Oligocene ones and the Santa Rosa ?Eocene/Oligocene one. These new data document a poorly known period in Western Amazonia, in providing a sedimentological record most likely documenting the late middle Eocene, the late Eocene and the earliest Oligocene interval. Furthermore, the existence of a long temporal hiatus between 
Canaanimys-Cachiyacuy-Potamotrygon ucayalensis assemblages (Contamana, Pozo Formation; Balsayacu; lower levels at Juanjui) and Eoincamys-yielding faunas (Santa Rosa; Tarapoto/Shapaja; uppermost levels at Juanjui) is demonstrated in a single continuous and long-ranging stratigraphic section at Juanjui.

\section{EXTENDED ABSTRACT}

Today, rodents are the most diverse and speciose group of placental mammals. A significant part of that diversity is illustrated by hystricognathous rodents from South America: the caviomorphs (Caviomorpha Wood, 1955). This group - the fossil record of which so far extends back to the late middle Eocene - exhibits at least 40 million years of endemic evolution in the South American continent. Caviomorphs are usually divided into four superfamilies: Octodontoidea (spiny rats and their allies), Erethizontoidea (New World porcupines), Chinchilloidea (chinchillas and their allies), and Cavioidea (guinea pigs and their allies). From an evolutionary paleontological perspective, despite the incompleteness of the fossil record, the great diversity of extant caviomorphs is the result of several adaptive radiations, which occurred primarily during the Miocene, but also during the Paleogene. Indeed, just after their arrival on that isolated giant landmass, likely from Africa sometime during the middle Eocene (late Lutetian; Barrancan SALMA [South American Land Mammal Ages]), this group has rapidly diversified, as demonstrated by the great specific diversity observed in the few South American Oligocene localities known thus far. Deposits dating from the Tinguirirican SALMA (early Oligocene) and from the Deseadan SALMA (late Oligocene) of Argentinean and Chilean Patagonia, Bolivian Altiplano or Peruvian Amazonia, yield among the earliest representatives of the main extant superfamilies of Caviomorpha. If during the Eocene, stem caviomorph species were seemingly limited to low latitudes of the Neotropics and did not exhibit a wide range of body-sizes and morphologies, by contrast the Deseadan (even pre-Deseadan [Tinguirirican]) testifies of an important diversity of species, which spread over several latitudes, up to the Caribbean. The first caviomorph faunas are known by a majority of small and fragmented isolated teeth, which are brachydont and characterized by primitive buno-lophodont patterns, including straight crests and cristids. These morphological conditions contrast with those of taxa from Deseadan and Neogene localities showing lophodont patterns and teeth tending to be more hypsodont with a higher obliquity of the crests and cristids.

In this paper, we report and describe new rodent fossil remains discovered during our recent field missions (2015-2016) in Peruvian Amazonia, in a geographical area yielding two new stratigraphic sections located in the vicinity of the cities of Juanjui and Balsayacu (San Martín Department, Peru). The fossil material consists only of isolated teeth recovered after wet-screening of sediments of several new localities, but it documents different species revealing a marked morphological diversity in terms of dental patterns (occlusal structures). The analysis of the occlusal morphologies of cheek teeth indicate the occurrence of basal caviomorphs (such as Cachiyacuy, Canaanimys, and Eoespina, genera so far known from late middle Eocene deposits of Contamana, Peruvian Amazonia) in levels situated in the lower part of the studied stratigraphic sections (Juanjui: TAR-45, 46, 47; Balsayacu: TAR-55, 55bis, 56). More derived taxa (such as Eoincamys, known from early Oligocene deposits of Tarapoto/Shapaja and ?late Eocene/early Oligocene deposits of Santa Rosa, Peruvian Amazonia) occur only in upper levels (Juanjui: TAR-49, 50). These taxonomic observations are corroborated by the study of the enamel microstructure, which was performed on incisor fragments found in the same localities having yielded the rodent cheek teeth. Indeed, the basal levels (Juanjui: TAR-45, 46, 47; Balsayacu:TAR-55, 55bis, 56) contain incisors whose enamel has a microstructure characterized by subtypes 1, 1-2 or 2 of multiserial Hunter-Schreger bands (HSB), whereas the more recent levels (Juanjui: TAR-49, 50) yield enamel incisors with the subtype 2-3 of multiserial HSBs, a more derived and more resistant enamel microstructure.

Based on these considerations, the rodent-bearing localities of the Juanjui/Balsayacu area seem to be intermediate in age between the Contamana Eocene localities and the Tarapoto/Shapaja Oligocene ones and the Santa Rosa ?late Eocene/Oligocene one. These new data document a poorly known period in Western Amazonia, in providing a sedimentological record most likely documenting the late middle Eocene, the late Eocene and the earliest Oligocene interval. Furthermore, the existence of a long temporal hiatus between Canaanimys-Cachiyacuy-Potamotrygon ucayalensis assemblages (Contamana, Pozo Formation; Balsayacu; lower levels at Juanjui) and Eoincamys-yielding faunas (Santa Rosa; Tarapoto/Shapaja; uppermost levels at Juanjui) is demonstrated in a single continuous and long-ranging stratigraphic section at Juanjui. The sedimentological section of Juanjui is therefore of great interest for the history of Neotropical ecosystems, as it documents a time window before and around the Eocene/Oligocene transition (EOT), which has so far been poorly or not documented in the low latitudes of South America. This period is characterized by a significant global cooling, associated with severe faunal rearrangements at the global scale (faunal turnovers, extinctions, dispersals). Accordingly, the identification in Peruvian Amazonia of a fossiliferous sedimentological series framing the EOT should, for the first time, allow for an assessment of the impact of this global event on the faunas and floras from the Neotropical regions. 


\section{INTRODUCTION}

Parmi les mammifères placentaires, les rongeurs représentent le groupe le plus diversifié (par ex: Wilson \& Reeder 2005). $\mathrm{Au}$ sein de cet ordre, se distingue un groupe endémique d'Amérique du Sud et Centrale: les caviomorphes (Caviomorpha Wood, 1955). Ces rongeurs appartiennent au clade des Hystricognathi Tullberg, 1899, et sont parmi les mammiferes les plus abondants du continent sud-américain (Wilson \& Reeder 2005). Les caviomorphes sont couramment divisés en quatre superfamilles: Octodontoidea Waterhouse, 1839, Erethizontoidea Bonaparte, 1845, Chinchilloidea Bennett, 1833 et Cavioidea Fischer de Waldheim, 1817. Les formes actuelles présentent une grande diversité écologique et taxinomique (par ex. : Mares \& Ojeda 1982; Patton et al. 2015) qui serait le résultat de plusieurs évènements de radiation pendant le Néogène et le Pléistocène (Candela et al. 2012; Vucetich et al. 2010, 2015a). Cependant l'histoire évolutive du groupe ne se limite pas aux derniers 20 millions d'années (Ma) mais remonte au moins à la fin de l'Éocène moyen. En effet, les plus anciens restes fossiles connus de ce groupe ont été récemment identifiés en Amazonie péruvienne (région de Contamana, Département du Loreto; Antoine et al. 2012; Boivin et al. 2017a), dans des dépôts datant de la fin de l'Éocène moyen (c. $41 \mathrm{Ma}$; Barrancien SALMA [South American Land Mammal Age]). L'histoire évolutive des caviomorphes s'échelonne ainsi sur plus de 40 millions d'années sur le continent sud-américain. Après leur arrivée probable à partir du continent africain au cours de l'Éocène moyen (par ex.: Antoine et al. 2012; Fabre et al. 2013, 2015; Marivaux et al. 2014; Barbière \& Marivaux 2015), dans un environnement visiblement propice, ce groupe de rongeurs hystricognathes s'est rapidement diversifié. En effet, des représentants des quatre superfamilles de caviomorphes sont déjà documentés dans le Paléogène, principalement d'Argentine (Tinguiriricien SALMA et Déséadien SALMA; Wood 1949; Vucetich et al. 2010), du Chili (Tinguiriricien; Bertrand et al. 2012), de Bolivie (Déséadien; Lavocat 1976), et d'Amazonie péruvienne (Tinguiriricien-Déséadien SALMA; Frailey \& Campbell 2004; Boivin 2017; Boivin et al. 2017b, 2018, 2019a). Les plus anciens représentants des caviomorphes (Barrancien SALMA; Contamana) sont exclusivement connus aux basses latitudes et montrent une faible diversité morphologique (petite taille, brachyodontie, buno-lophodontie et lophes fins et rectilignes; Antoine et al. 2012; Boivin et al. 2017a). En revanche, pour les périodes plus récentes (post-Tinguiriricien), on enregistre une bien plus grande diversité taxinomique et une extension considérable de l'aire de répartition géographique, allant de la Patagonie (Vucetich et al. 2015b) jusqu'aux Caraïbes (VélezJuarbe et al. 2014), avec des formes lophodontes et certaines montrant une tendance à l'hypsodontie et à l'obliquité des lophes (Wood \& Patterson 1959; Lavocat 1976; Boivin et al. 2017b).

Parmi les plus anciens sites à rongeurs, seuls ceux de Termas del Flaco (Chili; Wyss et al. 1993; Bertrand et al. 2012), de La Cantera (Argentine; Vucetich et al. 2010) et de Contamana
(Amazonie péruvienne; Antoine et al. 2012; Boivin et al. 2017a) précèdent le Déséadien et bénéficient d'une datation radiochronologique. D'autres sites comme Santa Rosa (Frailey \& Campbell 2004) ou Tarapoto/Shapaja (Boivin et al. 2018) d'Amazonie péruvienne ont été estimés comme préDéséadien (très certainement Tinguiriricien), sur la base de leurs assemblages fauniques, et partagent des ressemblances avec certains taxons décrits à CTA-27. La localité de Santa Rosa, initialement considérée comme d'âge Éocène supérieur (Frailey \& Campbell 2004), semble, à la lumière des récentes découvertes, être très proche de la transition ÉocèneOligocène, voire d'âge Oligocène basal (Antoine et al. 2016; Boivin et al. 2018).

Dans le présent article, nous rapportons et décrivons des restes inédits de rongeurs découverts à l'occasion de missions de terrain récentes (2015-2016) effectuées en Amazonie péruvienne, dans un secteur géographique situé à proximité des villes de Juanjui et de Balsayacu, dans le Département de San Martín (Fig. 1). Le secteur de Juanjui et de Balsayacu revêt un intérêt certain du fait de sa localisation quasi-équatoriale, dans la zone sub-andine du bassin du Huallaga (Hermoza et al. 2005; Roddaz et al. 2010). Sera ici mise en œuvre une approche systématique via l'étude de la morphologie dentaire et de la microstructure de l'émail des incisives, afin d'identifier le long des sections les différents taxons et de déterminer les types d'émail enregistrés. Seront ensuite abordées les implications biostratigraphiques par comparaison avec les rongeurs provenant d'autres gisements paléogènes, et notamment ceux d'Amazonie péruvienne (Contamana, Santa Rosa, et Shapaja/Tarapoto). Ce travail permettra ainsi d'apporter des précisions sur l'intervalle de temps enregistré dans cette série continentale et sur le degré évolutif des restes dentaires de rongeurs de cette période.

\section{MATÉRIEL ET MÉTHODES}

Les fossiles de rongeurs étudiés sont issus de huit localités (Fig. 1) situées le long de la route $5 \mathrm{~N}$ reliant Juanjui et Balsayacu, et longeant le Río Huallaga (Département de San Martín, Amazonie péruvienne). Cinq localités ont été découvertes aux abords de Juanjui (TAR-45, TAR-46, TAR-47, TAR-49 et TAR-50), et trois à proximité de Balsayacu (TAR-55, TAR55bis et TAR-56). La dénomination "TAR » des localités est utilisée par référence à Tarapoto, la capitale économique du Département de San Martín, où ont été menées les premières expéditions de terrain de l'équipe (2012-2013).

Le matériel fossile a été obtenu par lavage/tamisage de sédiments micro-conglomératiques peu consolidés, issus d'un paléo-système fluviatile. L'ensemble des localités a livré des restes dentaires, essentiellement sous forme de dents isolées, ou fragments de dents, à l'exception d'un fragment de mandibule portant deux molaires (MUSM-3391). D'autres restes fossiles ont été collectés, notamment des oogones de charophytes, des fragments d'exosquelettes de crustacés (crabes trichodactylidés), et des fragments de vertébrés aquatiques. Les fossiles de rongeurs étudiés et figurés dans ce papier sont déposés de façon 


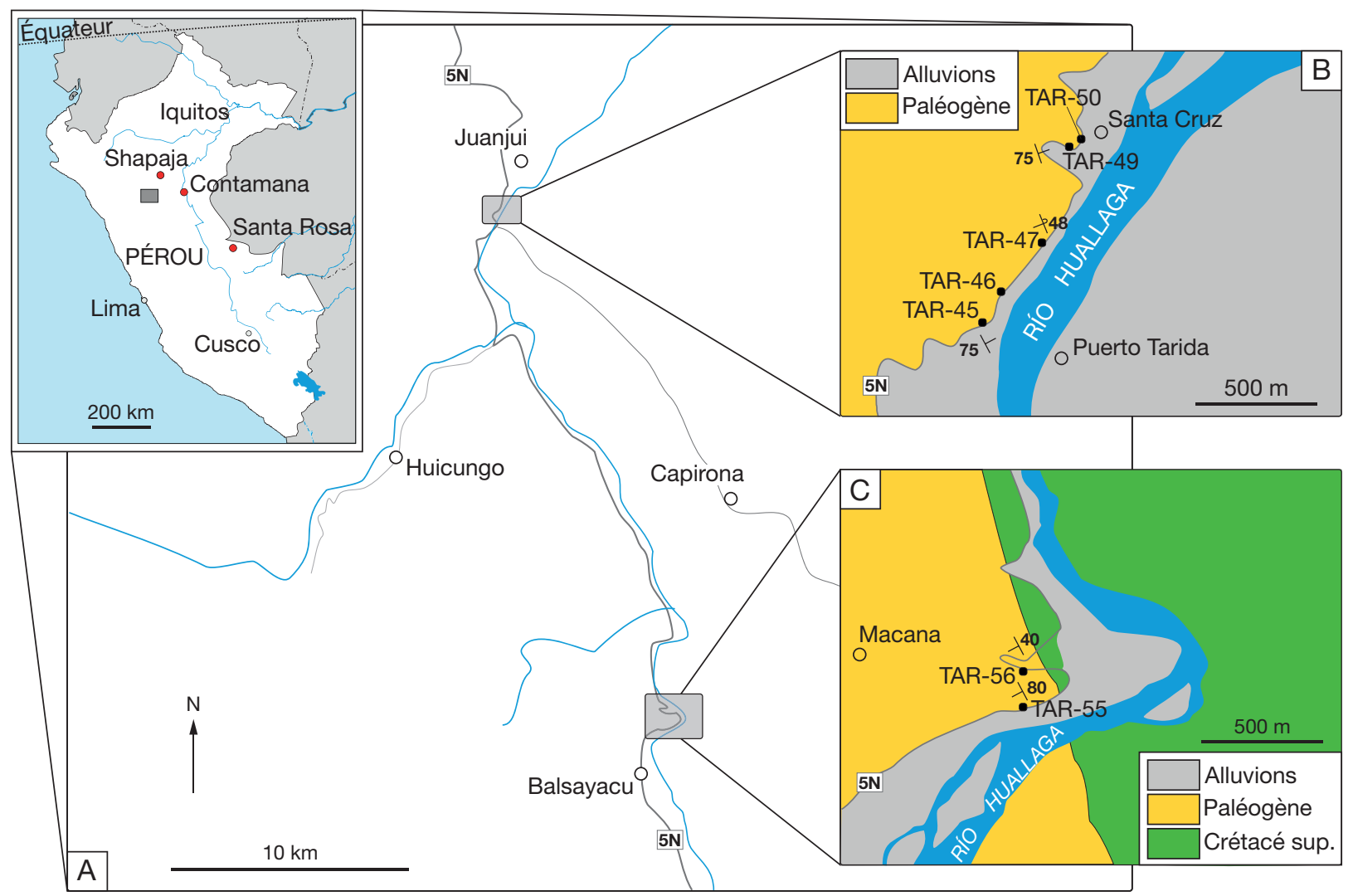

FIG. 1. - Localisation des sections et localités paléogènes de Juanjui et de Balsayacu, Département de San Martín, Pérou. Les localités à rongeurs paléogènes mentionnées dans le texte apparaissent en gras avec un disque rouge dans la carte du Pérou. A, carte de situation générale; B, carte géologique simplifiée de la section fossilifère de Juanjui et des localités à rongeurs; $\mathbf{C}$, carte géologique simplifiée de la section fossilifère de Balsayacu et des localités à rongeurs.

permanente dans les collections du Département de Paléontologie des Vertébrés du Museo de Historia Natural-Universidad Nacional Mayor San Marcos (MUSM) de Lima, Pérou.

Les restes dentaires de rongeurs ont été décrits en suivant la nomenclature établie par Boivin et al. (2017a, b, 2018) et Boivin \& Marivaux (2018), elle-même fondée sur les travaux de Wood \& Wilson (1936), Fields (1958) et Marivaux et al. (2004, 2014). Les dents isolées sont identifiées, regroupées et comparées sur la base de critères de taille, de hauteur de couronne et de patron occlusal.

La classification taxinomique est conforme à celle proposée par Boivin et al. (2019a), en particulier pour les taxons supra-génériques et leur hiérarchie. Dans ce manuscrit, le matériel rapporté à Eoespina a été comparé à l'ensemble des spécimens attribués à Eoespina woodi Frailey \& Campbell, 2004 et Eosachacui lavocati Frailey \& Campbell, 2004, pour lesquels une synonymie est supposée par Boivin et al. (2017a: 12, 13). Aussi, Eoespina fait référence ici à Eoespinal Eosachacui sensu Boivin et al. (2017a).

Les valeurs des dimensions des dents jugales (Tableau 1) ont été obtenues à l'aide d'un mesuroscope Nikon 10. Les valeurs de longueur mésiodistale (MD) et labiolinguale (LL), sont données en millimètres $(\mathrm{mm})$. Chaque prise de mesure a été répétée dix fois. Les dents présentées sur les Figures 3 et 4 ont été photographiées à l'aide d'un microscope électronique à balayage (MEB) HITACHI S 4000 .
Des analyses de microstructure de l'émail des incisives ont été réalisées sur 19 fragments de spécimens isolés, récoltés dans la plupart des localités, à l'exception de TAR-46, qui n'a livré que des dents jugales. Les mesures effectuées sur l'ensemble des incisives sont présentées dans le Tableau 2. La préparation des échantillons (coupes et digestions ménagées) a été opérée d'après le protocole suivi par Tabuce et al. (2007). Tous les spécimens ont été inclus dans de la résine époxy et poncés transversalement puis longitudinalement à l'aide d'un lapidaire équipé de disques de granulométries différentes (grain 180 à 4000). Les sections transversales des incisives ont été représentées selon Martin (2004: fig. 2). Pour rendre visibles les détails de la microstructure de l'émail, les échantillons polis ont été soumis à une attaque acide (acide phosphorique $37 \%$ ) pendant 30 secondes, puis rincés à l'eau distillée et enfin séchés. Les observations de la microstructure ont été réalisées au MEB (HITACHI S 4000) à différents grossissements (de × 300 à $\times$ 3000). La nomenclature utilisée pour la microstructure de l'émail est celle de Martin (1992, 1993) et Koenigswald \& Sander (1997). Les mesures effectuées suivent les protocoles de Martin (1992, 2004) et de Boivin et al. (2019b). Les mesures d'épaisseur de l'émail, d'angles des bandes d'Hunter-Schreger (HSB) avec la jonction émail/dentine (EDJ), d'angles entre les cristallites de la matrice inter-prismatique (IPM) et les cristallites de prismes, sont le résultat d'une moyenne de dix mesures, répétées sur les coupes. 
TABLEAU 1. - Attribution des restes dentaires des localités de Juanjui et de Balsayacu (Paléogène d'Amazonie péruvienne), et mesures correspondantes (mm). Abréviations: MD, mésiodistal; LL, labiolingual.

\begin{tabular}{|c|c|c|c|c|c|c|c|}
\hline $\begin{array}{l}\text { Numéros } \\
\text { collection }\end{array}$ & Localités & Loci & Fig. & État des specimens & Taxons attribués & (MD) & (LL) \\
\hline MUSM-3380 & TAR-45 & dP4 gauche & $4 S$ & incomplet et très abrasé & Caviomorpha gen. et sp. & - & - \\
\hline MUSM-3381 & TAR-45 & m2 droite & $4 \mathrm{R}$ & complet et peu abrasé & indet. 2 & 1,65 & 1,47 \\
\hline MUSM-3382 & TAR-46 & dP4 gauche & $3 \mathrm{~L}$ & subcomplet et abrasé & Cachiyacuy cf. contamanensis & 2,02 & 1,82 \\
\hline MUSM-3525 & TAR-47 & dP4 gauche & $4 \mathrm{C}$ & subcomplet et peu abrasé & Cachiyacuy aff. & 2,19 & 1,92 \\
\hline MUSM-3385 & TAR-47 & M gauche & $4 \mathrm{~A}$ & fragment abrasé & contamanensis & - & - \\
\hline MUSM-3386 & TAR-47 & M gauche & 4B & fragment peu abrasé & & - & - \\
\hline MUSM-3387 & TAR-47 & dp4 droite & $4 \mathrm{D}$ & $\begin{array}{l}\text { abrasé et partie antérieure } \\
\text { cassée }\end{array}$ & & - & - \\
\hline MUSM-3389 & TAR-47 & m1 droite & 4J & germe complet & & 1,76 & 1,88 \\
\hline MUSM-3390 & TAR-47 & m2 droite & $4 \mathrm{E}$ & germe complet & & 1,82 & 1,83 \\
\hline MUSM-3388 & TAR-47 & m2 gauche & $4 \mathrm{~L}$ & cassé mésiolabialement & & - & - \\
\hline MUSM-3393 & TAR-47 & m2 droite & $4 \mathrm{~K}$ & $\begin{array}{l}\text { peu abrasé mais cassée } \\
\text { mésiolingualement }\end{array}$ & & - & - \\
\hline MUSM-3391 & TAR-47 & $\begin{array}{l}\text { mandibule gauche } \\
\text { portant } \mathrm{m} 1 \text { et } \mathrm{m} 2\end{array}$ & $4 \mathrm{~F}-\mathrm{I}$ & subomplet mais abrasé & & $2,01 / 2,07$ & $1,9 / 2,06$ \\
\hline MUSM-3392 & TAR-47 & m3 droite & $4 \mathrm{M}$ & $\begin{array}{l}\text { subcomplet mais très } \\
\text { abrasé }\end{array}$ & & - & - \\
\hline MUSM-3394 & TAR-47 & m3 droite & $4 \mathrm{~N}$ & complet mais très abrasé & & 2,21 & 1,78 \\
\hline MUSM-3523 & TAR-47 & P4 droite & $3 \mathrm{H}$ & complet mais abrasé & Cachiyacuy aff. kummeli & 1,46 & 1,42 \\
\hline MUSM-3520 & TAR-47 & dp4 gauche & $3 \mathrm{~K}$ & complet mais abrasé & & 1,82 & 1,09 \\
\hline MUSM-3522 & TAR-47 & m3 droite & 31 & complet et peu abrasé & & 2,03 & 1,32 \\
\hline MUSM-3521 & TAR-47 & m3 droite & $3 \mathrm{~J}$ & complet mais abrasé & & 1,82 & 1,57 \\
\hline MUSM-3524 & TAR-47 & M2 gauche & $3 N$ & $\begin{array}{l}\text { abrasé et partie linguale } \\
\text { cassée }\end{array}$ & Eoespina sp. & - & - \\
\hline MUSM-3384 & TAR-47 & M droite & 30 & $\begin{array}{l}\text { peu abrasé mais partie } \\
\text { linguale cassée }\end{array}$ & & - & - \\
\hline MUSM-3383 & TAR-47 & M droite & $3 \mathrm{M}$ & complet mais abrasé & & 1,59 & 1,83 \\
\hline MUSM-3532 & TAR-49 & M gauche & $3 Q$ & fragment & Eoincamys sp. & - & - \\
\hline MUSM-3536 & TAR-50 & P4 gauche & $3 R$ & $\begin{array}{l}\text { subcomplet, hypocône } \\
\text { cassé }\end{array}$ & Eoincamys aff. pascuali & - & - \\
\hline MUSM-3537 & TAR-50 & M droite & $3 S$ & fragment abrasé & & - & - \\
\hline MUSM-3538 & TAR-50 & M droite & & éclat & & - & - \\
\hline MUSM-3535 & TAR-50 & m gauche & 3T & complet et peu abrasé & & 2,12 & 1,72 \\
\hline MUSM-3372 & TAR-55 & P4 gauche & $3 \mathrm{~A}$ & germe cassé en son centre & Cachiyacuy cf. kummeli & 1,81 & 1,31 \\
\hline MUSM-3371 & TAR-55 & M3 gauche & 40 & complet et peu abrasé & Canaanimys aff. maquiensis & 1,68 & 1,81 \\
\hline MUSM-3379 & TAR-55bis & M3 gauche & $4 \mathrm{P}$ & fragment abrasé & & - & - \\
\hline MUSM-3378 & TAR-55bis & M1 gauche & 3D & subcomplet et très abrasé & Cachiyacuy cf. kummeli & 1,69 & 1,78 \\
\hline MUSM-3377 & TAR-55bis & M2 droite & $3 C$ & $\begin{array}{l}\text { abrasé et cassé } \\
\text { postérieurement }\end{array}$ & & - & - \\
\hline MUSM-3376 & TAR-55bis & M2 gauche & 3B & subcomplet et abrasé & & - & - \\
\hline MUSM-3373 & TAR-55bis & m1 gauche & $3 \mathrm{E}$ & complet et peu abrasé & & 1,62 & 1,68 \\
\hline MUSM-3374 & TAR-55bis & m2 droite & $3 G$ & complet et peu abrasé & & 1,73 & 1,85 \\
\hline MUSM-3375 & TAR-55bis & $\mathrm{m} 2$ droite & $3 F$ & $\begin{array}{l}\text { très abrasé et cassé } \\
\text { antérolingualement }\end{array}$ & & - & - \\
\hline MUSM-3369 & TAR-56 & m droite & $3 P$ & $\begin{array}{c}\text { cassé antérieurement et } \\
\text { abrasé buccalement }\end{array}$ & cf. Eoespina sp. & - & - \\
\hline MUSM-3370 & TAR-56 & m3 droite & $4 Q$ & fragment & $\begin{array}{l}\text { Caviomorpha gen. et sp. } \\
\text { indet. } 1\end{array}$ & - & - \\
\hline
\end{tabular}

\section{CONTEXTE GÉOLOGIQUE}

La région d'intérêt (Fig. 1) est située dans le bassin d'avant-pays nord-péruvien, et plus précisément dans la zone sub-andine du bassin du Río Huallaga (Hermoza et al. 2005; Roddaz et al. 2010; Eude et al. 2015; Hurtado et al. 2018). Les sections paléogènes de Juanjui (Fig. 1B) et de Balsayacu (Fig. 1C) sont distantes d'environ $25 \mathrm{~km}$, sans continuité d'affleurement ni corrélation stratigraphique évidente a priori entre les deux zones, notamment à partir des éléments de la carte géologique (Sánchez Fernández \& Herrera Tufino 1998). En effet, si elles sont toutes deux constituées d'ensembles détritiques orientés N150 et à pendage moyen à fort, ces ensembles sont en revanche attribués respectivement aux formations Pozo (supposément Éocène; section de Balsayacu) et Chambira-Ipururo (supposément Oligocène-Miocène; section de Juanjui), mais jusqu'alors sans contrôle biostratigraphique ni contrainte chronostratigraphique formels (voir discussion dans Antoine 

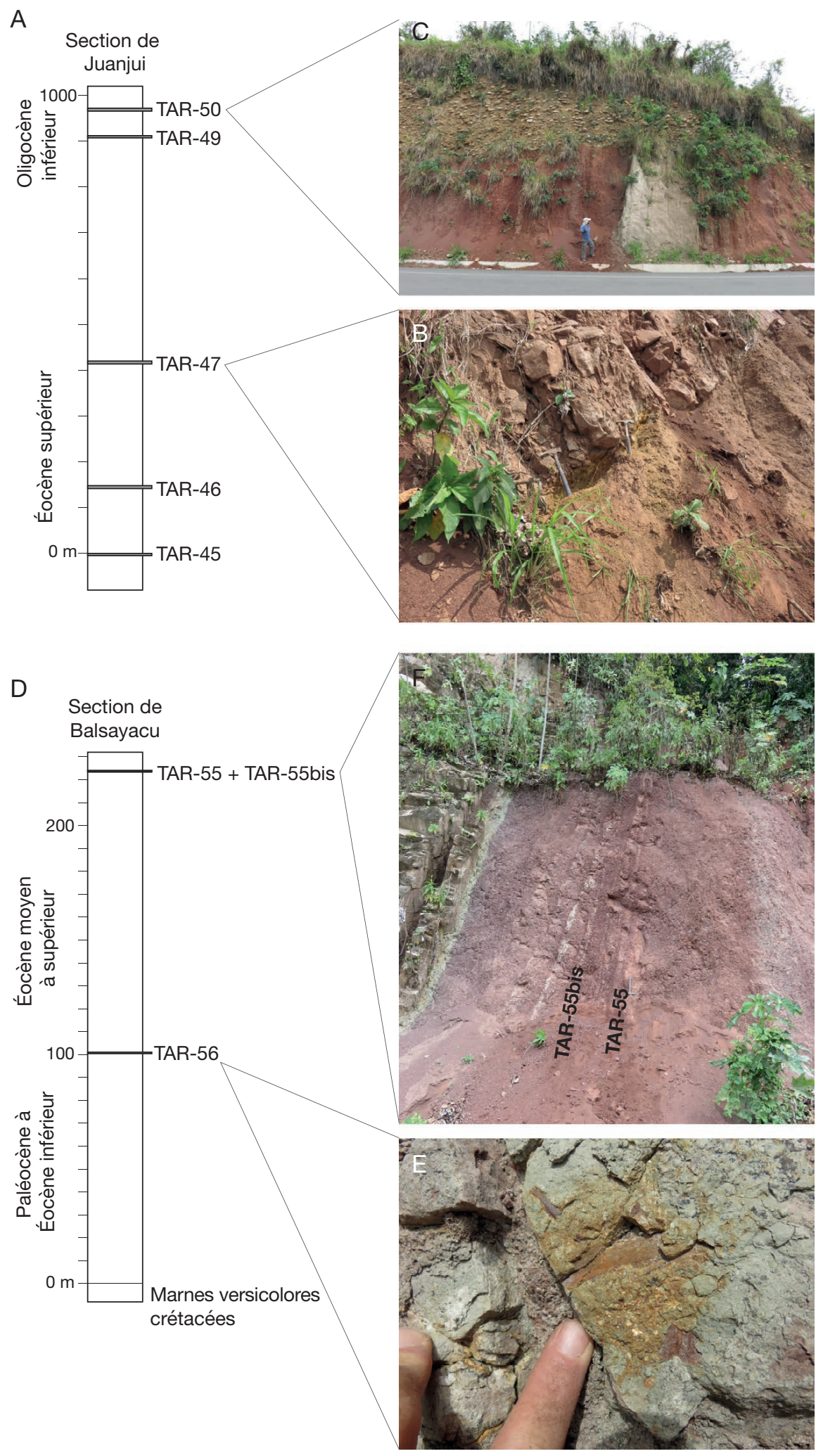

FIG. 2. - Sections stratigraphiques et positions des localités fossilifères de Juanjui et de Balsayacu. A-C, section de Juanjui : A, position stratigraphique respective des localités fossilifères TAR-45, TAR-46, TAR-47, TAR-49 et TAR-50, attribuées à l'Éocène supérieur et à l'Oligocène inférieur; B, détail des microconglomérats peu consolidés à matrice jaune de TAR-47; C, vue d'ensemble de TAR-50, situé à la base d'un chenal gréso-conglomératique intercalé dans des siltites rouges de plaine d'inondation. Les conglomérats pliocènes-pléistocènes sub-horizontaux de la formation Juanjui recouvrent la série paléogène avec une spectaculaire discordance angulaire. D-F, section de Balsayacu: $\mathbf{D}$, position stratigraphique respective des localités TAR-56, TAR-55 et TAR-55bis, attribuées à l'Éocène moyen-supérieur; $\mathbf{E}$, détail du microconglomérat peu consolidé de TAR-56, avec de nombreux restes végétaux oxydés (bruns); $\mathbf{F}$, Vue de l'affleurement incluant TAR-55 et TAR-55bis. 
et al. 2016; Boivin et al. 2018). Les affleurements fossilifères correspondent dans les deux cas à des microconglomérats peu consolidés à nodules calcaires, galets mous et restes végétaux oxydés, organisés en chenaux lenticulaires d'épaisseur décimétrique (TAR-55) à métrique (TAR-56) et d'origine fluviale à fluvio-deltaïque. Ces chenaux, seulement observés dans les remplissages d'âge éocène-oligocène inférieur dans les bassins de l'Ucayali et du Huallaga (Antoine et al. 2016; Klaus et al. 2017) y alternent avec des niveaux silteux rouges azoïques de grande épaisseur, correspondant à des dépôts de plaine d'inondation.

La section de Juanjui décrite ici est épaisse d'un millier de mètres (Fig. 2A). Le niveau le plus bas ayant livré des rongeurs est TAR-45 $\left(75^{\circ} \mathrm{E}-\mathrm{NE}\right)$. Il est surmonté parTAR-46 $\left(75^{\circ} \mathrm{E}-\mathrm{NE}\right)$ et TAR-47 ( $48^{\circ} \mathrm{O}-\mathrm{SO}$, série inversée), situés respectivement 140 et 420 mètres plus haut dans la série (Fig. 2B). Environ 490 mètres séparent ensuite TAR-47 de TAR-49 (75E-NE), et TAR-50 $\left(75^{\circ} \mathrm{E}-\mathrm{NE}\right)$ couronne la série paléogène, 60 mètres au-dessus de TAR-49 (Fig. 2C). Les conglomérats fluviatiles sub-horizontaux de la formation pliocène-pléistocène Juanjui recouvrent cette série, avec une spectaculaire discordance angulaire (Fig. 2C).

La section de Balsayacu, beaucoup plus courte, inclut les niveaux fossilifères TAR-56, TAR-55 et TAR-55bis (Fig. 2D). La localité TAR-56 (40O-SO; Fig. 2E) est située environ 100 mètres au-dessus du sommet de la série crétacée (marnes versicolores) et 120 mètres au-dessous de TAR-55. Le niveau TAR-55bis est un chenal décimétrique surmontant TAR-55 d'environ 1,5 mètres, dans le même affleurement $\left(80^{\circ} \mathrm{O}-\mathrm{SO}\right.$; Fig. 2F).

\section{SYSTÉMATIQUE}

Ordre RODENTIA Bowdich, 1821

Infraordre HYSTRICOGNATHI Tullberg, 1899

Parvordre CAVIOMORPHA Wood, 1955

Super-famille indet.

Genre Cachiyacuy Antoine, Marivaux, Croft, Billet, Ganerød, Jaramillo, Martin, Orliac, Tejada, Altamirano, Duranthon, Fanjat, Rousse \& Salas Gismondi, 2012

\section{Cachiyacuy cf. kummeli}

(Fig. 3A-G; Tableau 1)

MATÉRIEL. - MUSM-3373, m1 gauche (Fig. 3E) ; MUSM-3374, $\mathrm{m} 2$ droite (Fig. 3G) ; MUSM-3375, m2 droite (Fig. 3F) ; MUSM3372, P4 gauche (Fig. 3A); MUSM-3378, M1 gauche (Fig. 3D); MUSM-3376, M2 gauche (Fig. 3B); MUSM-3377, M2 droite (Fig. 3C).

Localité. - TAR-55bis, Balsayacu, Département de San Martín, Pérou.

\section{DESCRIPTION}

La m1 (MUSM-3373; Fig. 3E) présente un contour subrectangulaire, caractérisé par un métaconide très anguleux et dominant. Cette molaire est tétralophodonte et non téni- odonte. Le protoconide et l'entoconide sont bien marqués tandis que l'hypoconide est d'apparence crestiforme, mais se distinguant encore du postérolophide. L'obliquité des cristides est faible et l'émail peu épais. Les vallées sont relativement profondes par rapport à la hauteur des cristides. Le métalophulide I est rectiligne mais discontinu. Il est composé du bras antérieur du protoconide et de celui du métaconide, lesquels se connectent centralement sur l'axe labiolingual. Un faible bourrelet d'émail, situé distalement sur le bras postérieur du métaconide, indique la présence d'un mésostylide. De ce dernier s'étend la deuxième cristide, sinueuse et composée d'un néomésolophide court qui rejoint le long bras postérieur du protoconide connecté à l'ectolophide. Le bras antérieur de l'hypoconide est bien marqué et orienté obliquement de l'hypoconide vers l'hypolophide. Le postérolophide rejoint l'entoconide à sa base, laissant une encoche ouvrant le métaflexide lingualement.

Les m2 (MUSM-3374 et 3375; Fig. 3F, G) présentent une architecture occlusale similaire à celle observée sur les $\mathrm{m} 1$, mais en différent par leur forme plus quadrangulaire et transverse.

La M1 MUSM-3378 (Fig. 3D) est brachyodonte, tétralophodonte, non téniodonte et présente un émail plus épais lingualement que labialement. Les cuspides sont bien définies et les lophes sont rectilignes et transverses. Seul le bras antérieur de l'hypocône est orienté obliquement et se connecte à la mure. Le postérolophe est épais et pourrait correspondre à un complexe métacône-métalophe-postérolophe (voir Boivin \& Marivaux 2018). Le mésostyle est marqué, et de ce dernier part un long mésolophe connecté au bras antérieur de l'hypocône via un mésolophule peu marqué et très court. Une crête accessoire connecte le mésolophe au postérolophe/complexe (métacône-métalophe-postérolophe), délimitant ainsi deux fossettes postérieures: la première est triangulaire, peu étendue et située labialement à l'hypocône, et la seconde est sub-ovale, plus étendue et longeant le mésolophe. Le protolophe labial est rectiligne, légèrement oblique, et relie le paracône de petite taille au protolophe lingual. Ce dernier ferme lingualement l'antérofossette. L'antérolophe, reliant le très large protocône au paracône, ferme l'antérofossette labialement.

Les M2 (MUSM-3376 et MUSM-3377; Fig. 3B, C) ont une morphologie occlusale similaire à celle de la M1, mais présentent un contour plus transverse et arrondi du fait de la courbure marquée de leur antérolophe et de leur postérolophe. Les cuspides sont bulbeuses à l'exception du protocône qui est crestiforme. Le mésostyle est bien marqué, occupant une position légèrement plus labiale que les deux autres cuspides labiales.

\section{COMPARAISONS}

Ce taxon diffère de Canaanimys maquiensis Antoine, Marivaux, Croft, Billet, Ganerød, Jaramillo, Martin, Orliac, Tejada, Altamirano, Duranthon, Fanjat, Rousse \& Salas Gismondi, 2012, par la tétralophodontie de ses M1-2 et la présence d'une deuxième cristide complète et robuste sur les molaires inférieures; de Mayomys confluens Boivin, Marivaux, Pujos, Salas-Gismondi, Tejada-Lara, Varas-Malca \& Antoine, 2018 par la présence d'un métaconide très anguleux sur les molaires 


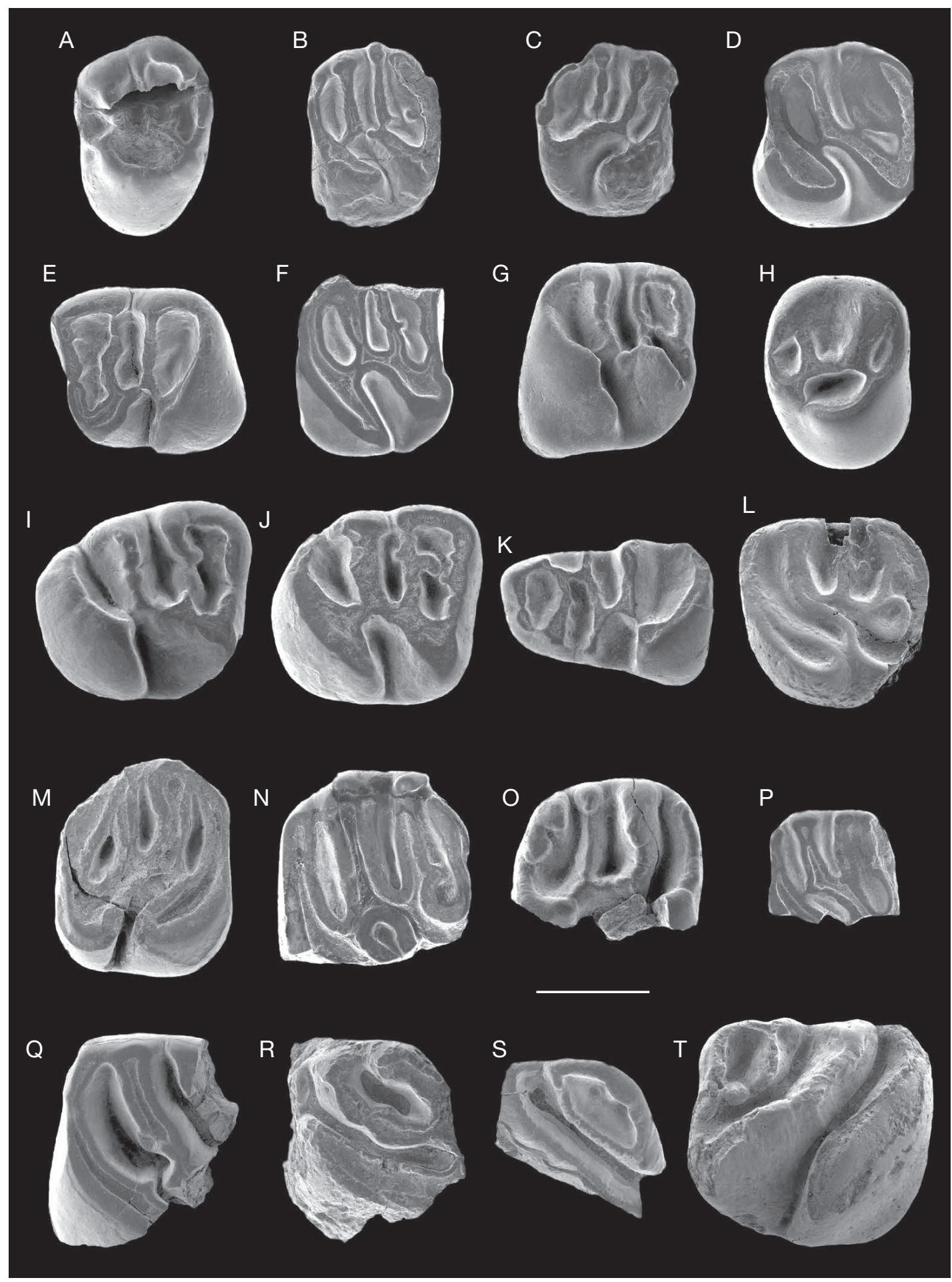

FIG. 3. - Images de microscopie électronique à balayage des dents jugales de caviomorphes fossiles de la région de Juanjui et de Balsayacu en vue occlusale: A-G, Cachiyacuy cf. kummeli (TAR-55bis): A, MUSM-3372, P4 gauche; B, MUSM-3376, M2 gauche; C, MUSM-3377, M2 droite; D, MUSM-3378, M1 gauche; E, MUSM-3373, m1 gauche; F, MUSM-3375, m2 droite; G, MUSM-3374, m2 droite; H-K, Cachiyacuy aff. kummeli (TAR-47): H, MUSM-3523, P4 droite; I, MUSM-3522, m3 droite; J, MUSM-3521, m3 droite; K, MUSM-3520, dp4 gauche; L, Cachiyacuy cf. contamanensis (TAR-46): MUSM-3382, dP4 gauche; M-O, Eoespina sp. (TAR-47): M, MUSM-3383, M1-2 droite; N, MUSM-3524, M2 gauche; O, MUSM-3384, M droite; P, cf. Eoespina (TAR-56): MUSM-3369, m droite; Q, Eoincamys sp. (TAR-49): MUSM-3532, M gauche; R-T, Eoincamys aff. pascuali (TAR-50): R, MUSM-3536, P4 gauche; S, MUSM-3537, M droite; T, MUSM-3535, m gauche. Échelle: $1 \mathrm{~mm}$.

inférieures; et de Cachiyacuy kummeli Antoine, Marivaux, Croft, Billet, Ganerød, Jaramillo, Martin, Orliac, Tejada, Altamirano, Duranthon, Fanjat, Rousse \& Salas Gismondi, 2012, par sa plus grande taille et la présence d'une connex- ion du postérolophide plus basse sur l'entoconide, lequel est moins élevé que chez ce dernier. Il est toutefois à rapprocher de cette dernière espèce, avec laquelle il présente de plus fortes ressemblances. 


\section{Cachiyacuy aff. kummeli \\ (Fig. 3H-K; Tableau 1)}

MATÉRIEL. - MUSM-3520, dp4 gauche (Fig. 3K) ; MUSM-3521, m3 droite (Fig. 3J); MUSM-3522, m3 droite (Fig. 3I) ; MUSM3523, $\mathrm{P} 4$ droite (Fig. 3H)

LoCALITÉ. - TAR-47, Juanjui, Département de San Martín, Pérou.

\section{DESCRIPTION}

La dp4 (MUSM-3520 ; Fig. 3K) est de petite taille et allongée mésiodistalement. Cette prémolaire brachyodonte présente un schéma occlusal pentalophodonte et non téniodonte. Le protoconide et l'entoconide sont bulbeux tandis que l'hypoconide et le métaconide ont une forme plus effilée. Le postérolophide est très courbé et ne rejoint pas l'entoconide, laissant le métaflexide largement ouvert lingualement. Le bras antérieur de l'hypoconide est aminci et sépare le métaflexide de l'hypoflexide. L'hypolophide est rectiligne et transverse, connecté à l'ectolophide distal et au bras antérieur de l'hypoconide. Sur le bord lingual de la dent, un petit mésostylide est présent. Ce dernier, étiré labiolingualement, s'étend labialement via un néomésolophide rectiligne, qui rejoint une cuspide labiale (mésoconide déplacé labialement ou néocuspide). Lingualement à ce dernier est présent un ectolophide mésial, orienté mésiolabialement. Le protoconide est distal par rapport au métaconide. Les deux cuspides sont reliées par un métalophulide I court et arqué. Le bras postérieur du protoconide, long et recourbé, rejoint le bord lingual de la dent, au niveau de l'extrémité distale du bras postérieur du métaconide. Cette deuxième cristide forme un métalophulide II. Une cristulide longitudinale le relie au néomésolophide.

Les molaires MUSM-3521 (Fig. 3J) et MUSM-3522 (Fig. 3I) sont identifiées comme des $\mathrm{m} 3 \mathrm{du}$ fait de leur forme caractéristique, montrant une compression labiolinguale de la partie distale du talonide. Ces dents sont brachyodontes, tétralophodontes et non téniodontes. Leurs hypoconide et métaconide sont crestiformes. Le protoconide et l'entoconide sont en revanche bulbeux. Le métaconide se prolonge distalement par un fort bras postérieur qui se connecte à un mésostylide bien marqué. Une profonde et étroite encoche sépare le mésostylide de l'entoconide (mésofossettide quasi fermée lingualement sur MUSM-3522). Mésialement, le métalophulide I sur MUSM-3521 est rectiligne et relie le bord mésiolabial du métaconide au bord mésiolingual du protoconide. Sur MUSM-3522 le métalophulide I apparait discontinu (présence d'une encoche étroite dans sa partie médiale). Le bras postérieur du protoconide est robuste et oblique dans sa partie labiale. Sa partie linguale est transverse et rejoint un court néomésolophide, la combinaison des deux cristides formant la deuxième cristide transversale. Sur MUSM-3521 (Fig. 3J), le métalophulide I et la deuxième cristide sont reliées par une néocristide courte et longitudinale qui tend à séparer l'antérofossettide en deux compartiments dans les stades d'usures les plus avancés. Cette dernière est à peine visible sur MUSM-3522 (Fig. 3I), présentant un état d'usure moindre. L'hypolophide est robuste et relie l'entoconide à l'ectolophide distal. L'hypoconide présente un bras antérieur très marqué, se connectant à l'ectolophide. Le postérolophide est robuste, très courbé et court, s'interrompant loin distalement de l'entoconide.

L'unique P4 (MUSM-3523 ; Fig. 3H) présente un contour subovale, lié à la formation de l'hypofossette par la fermeture de l'hypoflexus. Les cuspides sont bien définies à l'exception de l'hypocône, réduit. L'antérolophe s'étend du protocône à la base du paracône. Le protolophe est quant à lui complet et présente un fort bras lingual qui sépare la parafossette de l'hypofossette. Le protocône, orienté distomésialement, est crestiforme, et il forme le bord lingual de l'hypofossette. La mure est orientée distomésialement et se connecte antérieurement avec un mésolophule transverse qui rejoint un fort métacône oblique et mésiodistalement allongé. Cette configuration du métacône suggère la présence d'un mésostyle fusionné au métacône. Postérieurement, la mure se connecte à l'extrémité mésiale du bras antérieur de l'hypocône, lequel est court mais très épais. Enfin, le postérolophe s'étend jusqu'à l'extrémité linguale du métacône.

\section{COMPARAISONS}

Ce taxon differe de Canaanimys maquiensis par une forme plus trapue des $\mathrm{m} 3$ et la présence d'une deuxième cristide incomplète sur ces dernières; de Cachiyacuy contamanensis Antoine, Marivaux, Croft, Billet, Ganerød, Jaramillo, Martin, Orliac, Tejada, Altamirano, Duranthon, Fanjat, Rousse \& Salas Gismondi, 2012 (Contamana, Formation Pozo; Antoine et al. 2012; Boivin et al. $2017 \mathrm{a}$ ) et $C$. cf. contamanensis (TAR-46; voir ci-après) par la taille très petite de ses dents qui le rapprochent davantage de Cachiyacuy kummeli. Néanmoins, les molaires présentent des néoformations issues de la troisième crête, absentes chez $C$. kummeli. La taille des restes dentaires attribués à ce taxon est supérieure à celle des dents attribuées à $C$. cf. kummeli de TAR-55bis et ne permet pas de considérer formellement ces deux échantillons comme étant conspécifiques. Enfin, aucun locus dentaire ne permet une comparaison directe entre les patrons occlusaux de ces deux taxons. La prémolaire $(\mathrm{P} 4)$ differe des prémolaires supérieures d'Eobranisamys par l'absence de téniodontie et la présence d'une mure complète orientée transversalement; d'Eoincamys par la présence d'un fort protolophe lingual et l'absence de téniodontie; de Cachiyacuy contamanensis par l'absence de métalophe et une fusion complète du mésostyle avec le métacône; et de C. kummeli du fait de sa taille importante, d'une mure qui est complète et de la forme sub-ovale de son contour occlusal. Nous attribuons ces quatre dents à Cachiyacuy aff. kummeli.

\section{Cachiyacuy cf. contamanensis}

(Fig. 3L; Tableau 1)

MATÉRIEL. - MUSM-3382, dP4 gauche (Fig. 3L).

LoCALITÉ. —TAR-46, Juanjui, Département de San Martín, Pérou.

\section{DESCRIPTION}

Cette unique dent est brachyodonte, pentalophodonte et téniodonte. Les cuspides ont une allure relativement bul- 
beuse malgré la faible épaisseur de l'émail. Le mésostyle et l'hypocône restent visibles bien que partiellement cassés. Cette dent présente une forme trapézoïdale du fait de la constriction mésiodistale de sa partie linguale, entraînant une légère coalescence de l'hypocône avec le protocône, fermant ainsi l'hypoflexus lingualement. Le métacône est connecté au postérolophe courbé et au métalophe plus rectiligne. Du fait de l'usure, le métalophe est fusionné quasi complètement avec le postérolophe, et la postérofossette labiale est réduite à un petit îlot d'émail. Le métalophe rejoint également lingualement le bras antérieur de l'hypocône, et délimite ainsi, avec le postérolophe une postérofossette linguale circulaire qui est plus étendue que la postérofossette labiale. Le bras antérieur de l'hypocône se prolonge mésialement via la mure, laquelle est en continuité avec le protolophe labial, légèrement oblique. Labiocentralement s'observe un mésolophule transverse, qui relie la mure à la base du mésostyle. Cette dP4 est cassée labialement ce qui ne permet pas d'avoir accès ni à la morphologie du mésostyle, ni à la partie labiale de la troisième crête. Le mésolophule présente un petit éperon distal. Le protolophe ne présente pas de bras lingual le connectant au protocône, et l'hypoflexus est confluant avec l'antéroflexus. L'antérolophe est massif et arqué, et relie le protocône à l'extrémité labiomésiale du paracône.

\section{COMPARAISONS}

MUSM-3382 diffère de Cachiyacuy contamanensis et C. kummeli de CTA-27 du fait de sa téniodontie et de la présence d'un protocône jumelé avec l'hypocône; de Mayomys confluens du fait de sa taille bien supérieure à celle de ce dernier, de sa forme trapue, de sa pentalophodontie et téniodontie, ainsi que de la présence d'une troisième crête apparaissant complète. La comparaison du patron occlusal de MUSM-3382 avec MUSM-3387 attribuée à Cachiyacuy aff. contamanensis (voir ci-dessous) montre une incompatibilité d'occlusion ne permettant pas d'assigner cette dP4 à ce dernier taxon, mais plutôt à un taxon rapproché (Cachiyacuy cf. contamanensis).

\section{Cachiyacuy aff. contamanensis (Fig. 4A-N; Tableau 1)}

MATÉRIEL. - MUSM-3387, dp4 droite incomplète (Fig. 4D); MUSM-3389, germe de m1 droite (Fig. 4J); MUSM-3390, germe d'une $\mathrm{m} 2$ droite (Fig. 4E); MUSM-3388, $\mathrm{m} 2$ gauche cassée mésiolabialement (Fig. 4L); MUSM-3393, fragment de $\mathrm{m} 2$ droite (Fig. 4K) ; MUSM-3394, m3 droite (Fig. 4N) MUSM-3392, m3 droite sub-complète (Fig. 4M) ; MUSM3391, fragment de mandibule préservant la $\mathrm{m} 1$ et la $\mathrm{m} 2$ gauche (Fig. 4F-I) ; MUSM-3525, dP4 gauche (Fig. 4C) ; MUSM-3385, fragment de M1-2 gauche (Fig. 4A); MUSM-3386, fragment de M1-2 gauche (Fig. 4B).

LOCALITÉ. — TAR-47, Juanjui, Département de San Martín, Pérou.

\section{DESCRIPTION}

MUSM-3387 (Fig. 4D) est la partie postérieure d'une dp4 de grande taille présentant des cuspides marquées, un patron non téniodonte et un émail peu épais. Le postérolophide est très courbé et relie l'entoconide à l'hypoconide. L'hypolophide est rectiligne et se connecte à un bras antérieur de l'hypoconide très marqué délimitant ainsi une grande métafossettide. L'ectolophide fait la connexion entre l'hypolophide et un renflement d'émail pouvant être un mésoconide très usé, situé postérieurement au protoconide. Opposé lingualement à ce renflement d'émail, est un fort mésostylide, duquel part un néomésolophide qui ne rejoint pas l'ectolophide. Une deuxième cristide orientée vers l'avant s'étend de ce mésostylide, mais semble s'interrompre avant de rejoindre le métalophulide I (partie marquante de la dent).

MUSM-3389 (m1; Fig. 4J) est brachyodonte et tétralophodonte. La hauteur des cristides et des cuspides délimite des vallées et flexi profonds. Les cuspides sont majoritairement crestiformes à l'exception de l'entoconide qui est petit mais bien cuspidé. Le métalophulide I ne rejoint pas le protoconide, laissant ainsi une ouverture mésiolabiale de l'antéroflexide. Un long néomésolophide s'étend d'un mésostylide à peine marqué, situé sur l'extrémité distale du bras postérieur du métaconide. Ce mésostylide est séparé de l'entoconide par une profonde ouverture linguale du mésoflexide. Le néomésolophide rejoint le court bras postérieur du protoconide. L'ectolophide (essentiellement distal) est peu marqué et se connecte à l'extrémité labiale de l'hypolophide. Il n'y a pas de développement du bras antérieur de l'hypoconide, la dent présente ainsi un patron téniodonte. Le postérolophide, dans la continuité de l'hypoconide, est très courbé et rejoint à la base de l'entoconide le bras postérieur de cette cuspide, fermant ainsi lingualement le flexide transverse issu de la confluence entre l'hypoflexide et le métaflexide.

MUSM-3390 (m2; Fig. 4E) présente une morphologie globale très similaire à celle des $\mathrm{m} 1$. Cependant elle diffère de ces dernières par la présence d'un métalophulide I qui s'étend jusqu'à la base linguale du protoconide. Une deuxième cristide s'étend du bras postérieur du protoconide à un mésostylide bien marqué et quasiment fusionné au bras postérieur du métaconide. Cette cristide est composée d'une partie linguale, le néomésolophide, qui est rectiligne et fait un pli orienté mésiolabialement à son extrémité labiale, et d'une partie labiale (branche linguale du bras postérieur du protoconide), laquelle est oblique et orientée mésio-lingualement. MUSM-3390 (Fig. 4E), MUSM-3393 (m2; Fig. 4K) et MUSM-3388 (m2 ; Fig. 4L), différent de MUSM-3389 (Fig. 4J) par la présence d'un ectolophide plus marqué et se connectant à l'hypolophide. Le bras antérieur de l'hypoconide est incomplet et une connexion entre l'hypoconide et l'ectolophide est possible à partir de stades d'usure avancés (i.e., pseudo-téniodontie). Le postérolophide est très courbé et sa hauteur diminue lors de sa connexion avec l'entoconide, fermant ainsi lingualement le métaflexide.

La $\mathrm{m} 1$ préservée sur le fragment de mandibule (MUSM3391 ; Fig. 4F-I) présente un aspect rectangulaire, tétralophodonte et est brachyodonte et non téniodonte. Elle est plus petite que la $\mathrm{m} 2$ qui lui succède. Les deux dents préservées 
sont usées ce qui permet de voir que l'émail est très épais. Les cuspides sont bien marquées à l'exception du métaconide qui semble crestiforme malgré une cassure dans cette région. Le métalophulide I est rectiligne et transverse. La deuxième cristide, qui s'étend d'un fort mésostylide au protoconide, est divisée en deux parties: un néomésolophide court situé lingualement formant un étranglement lors de sa connexion avec le mésostylide, et une branche labiale du bras postérieur du protoconide très robuste. Cette deuxième cristide présente une néocristide antérieure orientée distomésialement. Cette dernière sépare l'antérofossettide en deux compartiments. Le bras postérieur du protoconide rejoint un ectolophide court sur lequel s'insère l'hypolophide, ce dernier étant rectiligne et transverse. Le bras antérieur de l'hypoconide est présent et bien marqué en raison de l'usure. Le postérolophide est épais et légèrement courbé, et son extrémité linguale est connectée à l'entoconide. La m2 de MUSM-3391 est très similaire à la $\mathrm{m} 1$, mais differe en étant de plus grande taille et de contour général plus quadrangulaire. La néocristide observée sur la $\mathrm{m} 1$ est absente sur la $\mathrm{m} 2$.

Les m3 (MUSM-3394 et MUSM-3392; Fig. 4M, N) sont particulièrement usées, mais leur structure occlusale reste identifiable, permettant d'observer un patron tétralophodonte et non téniodonte. Ces dents présentent un émail très épais sur leur partie distale et sont plus étroites labiolingualement dans cette région, du fait du postérolophide court. Le métalophulide I est rectiligne. Le postérolophide est particulièrement arqué. À ce stade d'usure (particulièrement sur la MUSM3394), le mésostylide est toujours visible, connecté au bras postérieur du métaconide. L'hypolophide est légèrement oblique et en continuité avec le complexe ectolophide-bras postérieur du protoconide. La deuxième cristide transverse semble être une combinaison d'un néomésolophide et de la branche linguale du bras postérieur du protoconide. Ces deux cristides sont de longueur égale. À ce stade d'usure, la région de l'hypoconide correspond à un fort élargissement distal au départ de son court bras antérieur, précédant le postérolophide. Ce bras antérieur est très court, et connecté à l'hypolophide (patron non téniodonte).

La dP4 (MUSM-3525; Fig. 4C) présente un contour trapézoïdal du fait d'une légère extension mésiodistale de sa partie labiale. Elle est brachyodonte et non téniodonte. Les cuspides sont bien visibles et le mésostyle est également bien développé. L'antérolophe recourbé présente un bourrelet d'émail qui peut être considéré comme un antérostyle. Le métacône, très crestiforme, forme un complexe indissociable du postérolophe. Du métacône, s'observe un métalophe court et oblique, lequel effectue un coude orienté postérieurement pour se connecter au postérolophe. L'hypocône tend à être crestiforme. Son bras antérieur est épais, oblique (dirigé mésiolabialement), et se connecte à la fois à la mure, complète et légèrement moins oblique, et à un mésolophule. Ce dernier est transverse et relie la jonction mure/bras antérieur de l'hypocône au mésostyle. Le paracône est fracturé mais volumineux. Il est connecté à un protolophe épais, complet et légèrement oblique, ainsi qu'à l'antérolophe qui le relie à un fort protocône.
MUSM-3385 (Fig. 4A) et MUSM-3386 (Fig. 4B) sont des parties distolabiales de molaires supérieures. Bien que fragmentaires, elles sont de toute évidence pentalophodontes du fait de la présence d'une troisième et quatrième crêtes bien développées (cf. description ci-dessous), et présentent un émail épais. Les cuspides sont marquées et les crêtes sont rectilignes et transverses. Le paracône est relié à une mure épaisse via un protolophe labial très développé (MUSM3386). Une troisième crête s'étend d'un mésostyle bien défini à la jonction entre la mure et le bras antérieur de l'hypocône. Cette crête semble être composée d'un long mésolophe auquel se connecte lingualement un court mesolophule, mais l'usure ne permet pas de statuer sur cette structure de manière certaine. Le métalophe est bien défini et long. Il part du métacône et se connecte distalement, dans la partie visiblement centrale du postérolophe. Seul un fragment labial de l'antérolophe est visible sur la MUSM-3386, cette crête étant complètement manquante sur la MUSM-3385. La présence d'une écaille de protolophe lingual sur la MUSM-3386 suggère que cette dent n'était probablement pas téniodonte.

\section{COMPARAISONS}

Cet échantillon diffère des dents de Canaanimys maquiensis, Mayomys confluens et Cachiyacuy kummeli par ses plus grandes dimensions; de Canaanimys par la présence d'un métalophe orienté postérieurement et se connectant sur le postérolophe labialement à l'hypocône; de Cachiyacuy kummeli par la présence d'un métalophe complet, d'un métacône séparé du postérolophe par une profonde encoche, et par la présence de lophes moins graciles. Certaines des molaires inférieures décrites ici présentent une variabilité dans le développement du bras antérieur de l'hypoconide, ce qui confère à certains spécimens une structure non téniodonte et à d'autres une structure pseudo-téniodonte à téniodonte. De manière générale la téniodontie n’a pas été observée sur les molaires inférieures de C. contamanensis (Antoine et al. 2012; Boivin et al. 2017a), bien qu'une pseudo-téniodontie soit décrite sur quelques spécimens attribués à $C$. cf. contamanensis morphe 1 (Boivin et al. 2017a). La dP4 décrite ici diffère de celle attribuée à $C$. cf. contamanensis de TAR-46 par un contour occlusal rectangulaire (allongé mésiodistalement) plutôt que quadrangulaire. La morphologie occlusale des dents inférieures se rapproche de celle des dents du genre Cachiyacuy, malgré l'existence de cas de pseudo-téniodontie observés sur certains spécimens. Les fragments de molaires supérieures décrits ici, par leur pentalophodontie (antérolophe, protolophe, mésolophe + mésolophule, métalophe et postérolophe) et leur apparente non téniodontie, pourraient également être compatible avec un patron Cachiyacuy, malgré la présence d'une connexion métalophe-postérolophe marquée. Pour toutes ces raisons, nous attribuons pour l'heure ce matériel au genre Cachiyacuy et le rapprochons notamment de l'espèce contamanensis (i.e, Cachiyacuy aff. contamanensis). L'acquisition de matériel supplémentaire documentant ce taxon devrait permettre de formaliser sa taxinomie. 


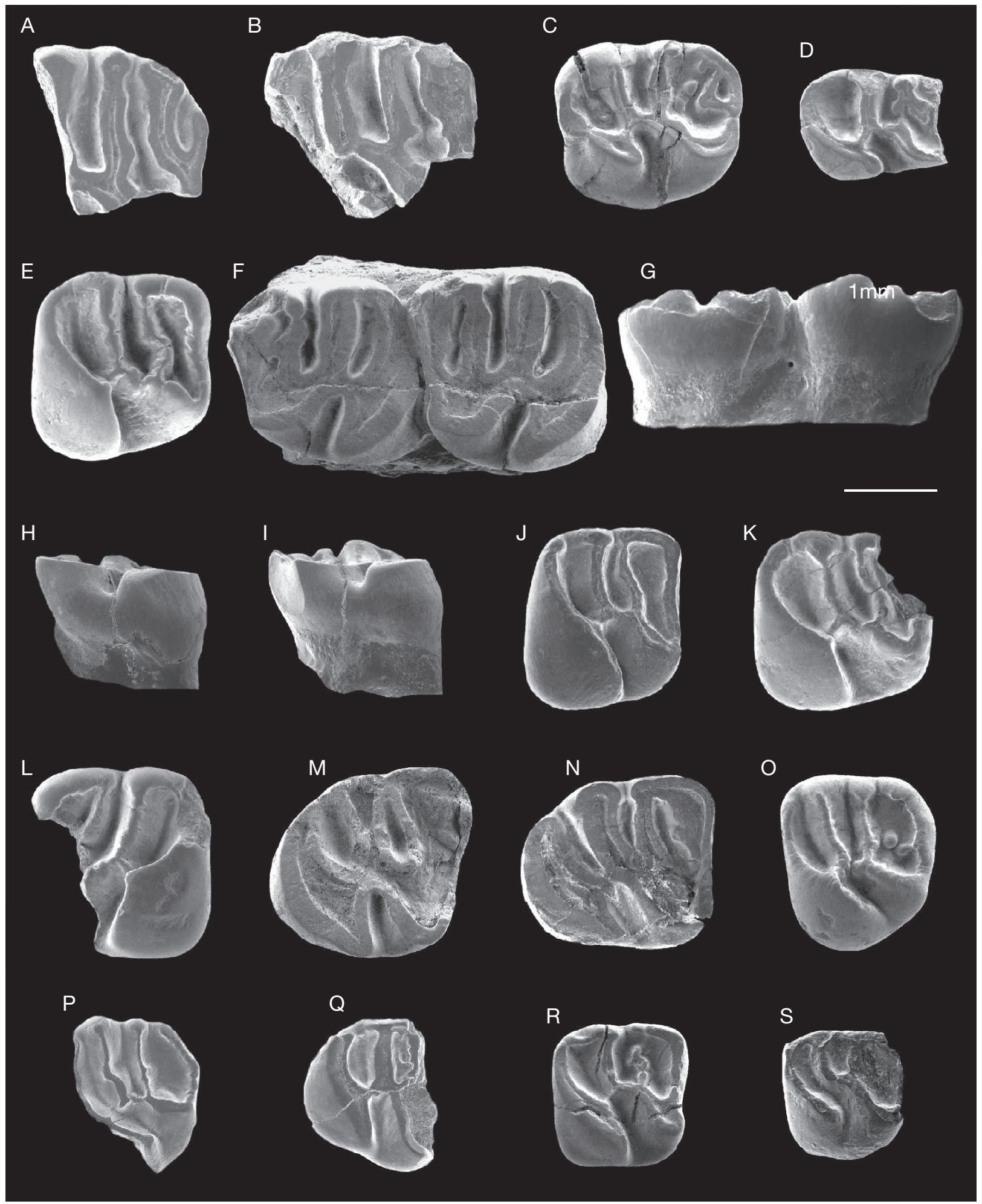

FIG. 4. - Images de microscopie électronique à balayage des dents jugales de caviomorphes fossiles de la région de Juanjui et de Balsayacu. A-F, J-S, vue occlusale; G, vue labiale; H-I, vue labiale. A-N, Cachiyacuy aff. contamanensis (TAR-47): A, MUSM-3385, M gauche; B, MUSM-3386, M gauche; C, MUSM-3525, dP4 gauche; D, MUSM-3387, dp4 droite; E, MUSM-3390, m2 droite; F-I, MUSM-3391, fragment de mandibule gauche portant m1 et m2; F-G, m1-2; H, m1; I, m2 ; J, MUSM-3389, m1 droite; K, MUSM-3393, m2 droite; L, MUSM-3388, m2 gauche; M, MUSM-3392, m3 droite; N, MUSM-3394, m3 droite. O-P, Canaanimys aff. maquiensis (TAR-55/ TAR-55bis): O, MUSM-3371, M3 gauche; P, MUSM-3379, M3 gauche. Q Caviomorpha gen. et sp. indet. 1. (TAR-56): MUSM-3370, m3 droite. R-S, Caviomorpha gen. et sp. indet. 2 (TAR-45): R, MUSM-3381, m2 droite; S, MUSM-3380, dP4 gauche. Échelle: 1 mm. 


\section{Genre Canaanimys}

Antoine, Marivaux, Croft, Billet, Ganerød, Jaramillo, Martin, Orliac, Tejada, Altamirano, Duranthon, Fanjat, Rousse \& Salas Gismondi, 2012

\section{Canaanimys aff. maquiensis}

(Fig. 4O, P; Tableau 1)

MATÉRIEL. - MUSM-3371, M3 gauche complète (Fig. 4O); MUSM-3379, fragment de M3 gauche (Fig. 4P).

LOCALITÉS. - TAR-55 \& TAR-55bis, Balsayacu, Département de San Martín, Pérou.

\section{DESCRIPTION}

MUSM-3371 (Fig. 4O) est brachyodonte, tétralophodonte et non téniodonte. Elle présente un contour arrondi ainsi que des lophes grêles, peu épais. Toutes les crêtes sont transverses, à l'exception du bras antérieur de l'hypocône, dans la continuité de ce dernier. Le métacône est petit et sensiblement égal en taille au mésostyle. La molaire présente un protocône et un hypocône crestiformes. Le postérolophe relie l'hypocône à la base du métacône. Il n'y a pas de métalophe dans la partie distolabiale de la dent, mais on peut noter la présence d'une postérofossette très ouverte dans laquelle s'individualisent des crestules d'émail, lesquelles peuvent être interprétées comme des néoformations ou des vestiges du métalophe. Le bras antérieur de l'hypocône, très développé, s'étend jusqu'à rejoindre une troisième crête transverse qui semble être un mésolophule, s'arrêtant à la base du mésostyle. De plus, la dent ne possède qu'une mure très peu marquée, sous la forme d'un éperon situé à l'extrémité mésiale du bras antérieur de l'hypocône. Cet éperon est à peine connecté au protolophe. Ce dernier est complet et épais, empêchant la confluence de l'hypoflexus avec le paraflexus. L'antérolophe est courbé et peu élevé; il s'étend du protocône à la base du paracône. Le paraflexus et le mésoflexus mésial sont profonds et étroits montrant la proximité des lophes sur la partie antérieure de la dent.

MUSM-3379 (Fig. 4P) est cassée antérieurement mais présente un patron occlusal assez similaire à celui de MUSM3371. À l'inverse du paracône bien développé, le métacône est réduit à une petite protubérance à l'extrémité labiale du postérolophe. Le mésostyle est bien développé. Le protolophe labial est robuste et non rectiligne. Le protolophe lingual est matérialisé par deux éperons d'émail (i.e., pseudo-téniodontie): le premier haut, épais et directement issu du protolophe labial et le second très fin et très bas reliant le premier éperon au protocône. Ce dernier se connecte à une mure incomplète et très basse. Du mésostyle part une troisième crête étroite qui semble être un mésolophe, se connectant au bras antérieur de l'hypocône. La présence d'une encoche entre le métacône et le mésostyle ouvre le flexus postérieur labialement.

\section{COMPARAISONS}

Ce matériel est rapproché du genre Canaanimys (seulement connu par l'espèce type C. maquiensis à CTA-27; Antoine et al. 2012; Boivin et al. 2017a), car il présente des crêtes étroites, transverses, des crestules pouvant être assimilées à un métalophe vestigial dirigé antérieurement ainsi qu'un grand flexus postérieur. Il diffère de ce dernier par la présence d'une téniodontie moins marquée ainsi que d'un métalophe incomplet. Il diffère également de Cachiyacuy par la taille de son flexus (ou fossette) postérieur et la présence d'une pseudo-téniodontie à téniodontie de la M3; d'Eobranisamys par l'absence de métalophe sur la M3 et l'aspect plus transverse de ses lophes. Compte tenu de leur patron morphologique, nous assignons ces spécimens à Canaanimys aff. maquiensis.

Genre Eoespina Frailey \& Campbell, 2004

Eoespina sp.

(Fig. 3M-O; Tableau 1)

MATÉRIEL. - MUSM-3383, M1-2 droite sub-complète (Fig. 3M); MUSM-3524, M2 gauche fragmentaire (Fig. 3N); MUSM-3384, M1-2 droite cassée lingualement (Fig. 3O).

LoCALITÉ. — TAR-47, Juanjui, Département de San Martín, Pérou.

\section{DESCRIPTION}

MUSM-3383 (Fig. 3M) et MUSM-3524 (Fig. 3N) sont tétralophodontes, brachyodontes, non téniodontes et possèdent un contour arrondi, ainsi que des crêtes étroites et hautes délimitant des fosses et flexi profonds. Les cuspides sont bien marquées et reliées par des crêtes rectilignes, à l'exception du bras antérieur de l'hypocône qui est oblique. Le protocône est bulbeux et présente une forte excroissance postérieure. La partie linguale du protolophe est moins développée que la partie labiale, mais sépare l'hypoflexus de la parafossette, ou du paraflexus à des stades d'usure moindres. Le paracône est relié à la mure par le protolophe labial, lequel est épais. Une troisième crête s'étend labiolingualement entre le mésostyle et la jonction mure/ bras antérieur de l'hypocône. Le métacône et le métalophe ne sont pas distincts. Cependant, la région postérolabiale du postérolophe est très épaisse, et témoigne probablement d'un complexe métalophe-métacône-postérolophe.

MUSM-3384 (Fig. 3O) présente un patron occlusal similaire à celui observé chez MUSM-3383 (Fig. 3M), mais l'ouverture labiale du paraflexus y est visible (usure moins avancée). Concernant la troisième crête transverse, un long mésolophule se connecte labialement à un très court mésolophe partant du mésostyle. Une tubérosité est située sur le postérolophe, lingualement au métacône, et pourrait indiquer la présence des vestiges d'un métalophe se connectant au postérolophe.

\section{COMPARAISONS}

MUSM-3383, MUSM-3524 et MUSM-3384 diffèrent des molaires supérieures de Cachiyacuy et de Canaanimys, par leur tétralophodontie et l'aspect arrondi de leur contour occlusal, caractères qui les rapprochent plutôt d'Eoespina; elles diffèrent néanmoins des molaires supérieures d'Eoespina par l'absence de fermeture labiale de la postérofossette et la présence d'un renflement plus marqué sur le postérolophe; elles diffèrent plus encore de celles de Mayomys confluens, par l'absence de fusion du mésostyle avec le métacône, et l'absence 
d'une fermeture labiale du postéroflexus. Le matériel n'est pas suffisant pour soutenir une identification spécifique, mais il peut être assigné avec confiance au genre Eoespina (sensu Boivin et al. 2017a) et laissé en nomenclature ouverte.

\section{Genre cf. Eoespina}

cf. Eoespina sp.

(Fig. 3P; Tableau 1)

MATÉRIEL. - MUSM-3369, fragment de molaire inférieure droite (Fig. 3P).

LOCALITÉ. - TAR-56, Balsayacu, Département de San Martín, Pérou.

\section{DESCRIPTION}

Malgré le caractère fragmentaire de cette unique dent (parties labiale et mésiale incomplètes), son contour semble carré ou légèrement transverse. Cette dent tétralophodonte, a une couronne basse et ne présente pas de téniodontie. La région mésiale de la dent est en partie cassée (notamment au niveau du métaconide) et ne laisse pas la possibilité de juger la configuration du métalophulide I. En revanche, distalement à cette cassure on distingue clairement la présence d'un néomésolophide épais, reliant le mésostylide à un large et court ectolophide. Le bras postérieur du protoconide est brisé. Cependant les éléments préservés montrent qu'il était oblique et épais, et rejoignait l'ectolophide et le néomésolophide. L'hypolophide est complet, le bras antérieur de l'hypoconide est épais et rejoint la partie linguale de l'hypolophide qui présente une légère obliquité.

\section{Comparaisons}

Ce spécimen est rapproché du genre Eoespina du fait de sa petite taille, de sa non-téniodontie, de la présence d'un patron tétralophodonte avec un bras postérieur du protoconide oblique et de celle d'un ectolophide court qui le différencient des genres Canaanimys et Cachiyacuy. Toutefois, du fait de l'état fragmentaire de cet unique spécimen (molaire inférieure), l'attribution générique de ce taxon est laissée en nomenclature ouverte (cf. Eoespina).

Super-famille CHINCHILlOIDEA Bennett, 1833 Genre Eoincamys Frailey \& Campbell, 2004

\section{Eoincamys aff. pascuali}

(Fig. 3R-T; Tableau 1)

MATÉRIEL. - MUSM-3535, m1 gauche complète (Fig. 3T); MUSM-3536, P4 gauche sub-complète (Fig. 3R); MUSM-3537, fragment de M1 ou M2 gauche (Fig. 3S) ; MUSM-3538, fragment de molaire supérieure droite (non figurée).

LOCALiTÉ. - TAR-50, Juanjui, Département de San Martín, Pérou.

\section{DESCRIPTION}

La molaire MUSM-3535 (Fig. 3T) est brachyodonte, tétralophodonte et téniodonte. La partie distale de sa couronne est plus élevée que sa partie mésiale. Elle présente des cristides très hautes et obliques ainsi que des vallées très profondes. Les cuspides sont très crestiformes dans leur ensemble, et relativement grêles. Le protoconide est présent mais peu distinct de son bras postérieur, lui-même connecté directement à l'hypolophide. L'ectolophide ne se distingue pas de l'hypolophide ni du bras postérieur du protoconide, avec lesquels il forme une cristide oblique centrale. Le métaconide est relié au protoconide par un métalophulide I court et fin. Une néocristide est dirigée mésiodistalement depuis le métalophulide I et se connecte à un court néomésolophide s'étendant depuis un petit mésostylide connecté à un long bras postérieur du métaconide. L'hypoconide crestiforme n'est pas distinct du postérolophide. Ces deux structures forment, avec l'excroissance antérieure de l'hypoconide, une longue cristide postérieure isolée de la cristide oblique centrale par une profonde confluence entre le métaflexide et l'hypoflexide.

La P4 MUSM-3536 (Fig. 3R) est trilophodonte et téniodonte. Les paracône et métacône sont renflés tandis que le protocône est large mais crestiforme. L'hypocône est quant à lui cassé mais fusionné à l'excroissance postérieure du protocône, fermant ainsi l'hypoflexus lingualement. Un parastyle petit et bas est présent à l'avant du paracône et développe de courtes crestules linguale et labiale. Sa crestule linguale est clairement séparée de l'antérolophe. Le protolophe est incomplet: seule est présente la partie labiale, laquelle est épaisse et se connecte avec le bras antérieur de l'hypocône (confluence entre l'hypoflexus et le paraflexus). La mure est absente. Une encoche entre le paracône et le métacône maintient le flexus postérieur ouvert labialement. Le postérolophe est courbé et épais.

MUSM-3537 (Fig. 3S) est un fragment de dent, correspondant à la partie distolabiale d'une M1 ou M2 supérieure gauche sur laquelle l'antérolophe et le protocône sont manquants. Ce fragment présente des lophes très hauts et obliques, délimitant des flexi et fossettes profondes. Les cuspides sont peu marquées et crestiformes. Le paracône développe un long bras postérieur séparé du bras antérieur du mésostyle par une fine encoche. Du paracône s'étend lingualement une crête oblique et massive résultant de la fusion du protolophe labial avec la mure et le bras antérieur de l'hypocône. L'absence de marques antérieures de connexion sur cette crête centrale montre que la dent était téniodonte. Le métacône est relié distolingualement au postérolophe, via une petite structure bulbeuse ("style » postérieur), et mésiolabialement au mésostyle via une longue crête, correspondant à un bras postérieur du mésostyle et/ou un bras antérieur du métacône. La troisième crête, oblique et parallèle à la crête centrale, connecte le mésostyle au postérolophe. Elle montre une encoche fine et peu profonde située aux abords du mésostyle, et paraît être ainsi constituée d'un court mésolophe partant du mésostyle et d'une longue crête partant du postérolophe (crête du postérolophe ?). Une structure très basse, pouvant être interprétée comme un reliquat de métalophe, relie la base du métacône à la troisième crête. 


\section{COMPARAISONS}

Cette dent diffère de celles d'Eobranisamys romeropittmanae Frailey \& Campbell, 2004, du fait de sa tétralophodontie; d'Eoincamys ameghinoi Frailey \& Campbell, 2004, par sa taille supérieure, du fait de ses crêtes orientées plus obliquement et de sa téniodontie; d'Eoincamys parvus Boivin, Marivaux, Pujos, Salas-Gismondi, Tejada-Lara, Varas-Malca \& Antoine, 2018, par sa taille largement supérieure et une connexion antérieure de la deuxième cristide sur le métalophulide I sur les molaires inférieures; d'Eoincamys valverdei Boivin, Marivaux, Pujos, SalasGismondi, Tejada-Lara, Varas-Malca \& Antoine, 2018, par une taille très légèrement plus petite et une couronne plus basse; d'Eoincamys pascuali Frailey \& Campbell, 2004, par un métacône encore distinct sur les molaires supérieures et une connexion antérieure de la deuxième cristide sur le métalophulide I sur les molaires inférieures. Toutefois, c'est de ce dernier taxon que MUSM-3537 s'approche le plus et nous l'attribuons de ce fait à Eoincamys aff. pascuali.

Eoincamys sp.

(Fig. 3Q; Tableau 1)

MATÉRIEL. - MUSM-3532, fragment de molaire supérieure gauche (Fig. 3Q).

LoCALITÉ. - TAR-49, Juanjui, Département de San Martín, Pérou.

\section{DESCRIPTION ET COMPARAISONS}

Ce fragment de dent est une partie antérieure d'une molaire supérieure gauche. L'émail est épais et les crêtes présentent une légère obliquité. Les cuspides sont marquées et forment l'élargissement terminal des crêtes. Du paracône descend un fort protolophe labial qui se connecte à une crestule cassée qui semble être la mure. Le protolophe lingual est incomplet, générant ainsi une pseudo-téniodontie de la dent (i.e., confluence sub-complète du paraflexus et de l'hypoflexus). Le protolophe lingual se décompose en un court bras postérieur du protocône et une autre crestule se développant depuis la mure. Ces deux structures semblent être connectées à leur base. L'antérolophe, épais et modérément recourbé, s'étend du protocône jusqu'au paracône, délimitant labialement le flexus antérieur (paraflexus + hypoflexus). Le bord labial de la dent présente un fort mésostyle, situé juste distalement au paracône. De ce style s'étend lingualement un court mésolophe. Nous attribuons ce fragment de dent à Eoincamys du fait de l'obliquité du protolophe et de la pseudo-téniodontie. Bien que MUSM3532 présente des crêtes obliques et des fosses profondes comme les spécimens de TAR-50 attribués à Eoincamys aff. pascuali, il diffère de ces derniers par une obliquité des crêtes moins importante. Compte tenu de son état fragmentaire et du manque de caractères spécifiques, nous rapportons ce spécimen à Eoincamys, mais le laissons en nomenclature ouverte.
Genre et espèce indet. 1

(Fig. 4Q; Tableau 1)

MATÉRIEL. - MUSM-3370, m3 droite incomplète (Fig. 4Q).

Localité. - TAR-56, Balsayacu, Département de San Martín, Pérou.

DESCRIPTION

MUSM-3370 est la partie distale d'une troisième molaire inférieure droite sur laquelle le métalophulide I, le métaconide et la partie mésiale du protoconide, de son bras postérieur et de la deuxième cristide transverse sont absents. La couronne est brachyodonte. L'entoconide est distinct, bulbeux et jumelé au mésostylide, fermant ainsi lingualement la mésofossettide. De ce stylide s'étend une deuxième cristide formée lingualement d'un néomésolophide long et rectiligne, et labialement d'une très courte cristulide orientée mésialement. De par son orientation et sa position très distale sur l'ectolophide, cette cristulide peut être interprétée comme un mésolophide. Elle se connecte de manière partielle au néomésolophide, générant ainsi une encoche. Le bras postérieur du protoconide est oblique et aligné avec l'ectolophide. L'hypolophide est épais et oblique, et se situe dans l'alignement avec le complexe bras postérieur du protoconide-ectolophide, formant ainsi une cristide diagonale orientée mesiolabialement-distolingualement. Du fait de l'état fragmentaire de MUSM-3370, seule une bordure distale d'émail d'une cristide rectiligne est présente dans la partie labiomésiale de la dent. Cette cristide inclurait à la fois le protoconide, son bras postérieur du protoconide et la partie mésiale de l'ectolophide. Le postérolophide est épais et courbé, et se connecte à l'hypolophide par la présence d'un bras antérieur de l'hypoconide très marqué et séparant l'hypoflexide du métaflexide (i.e., non téniodonte). Lingualement, le postérolophide se connecte à un éperon distal de l'entoconide. La configuration non téniodonte et la connexion postérolophide/ entoconide génèrent une métafossettide en forme de rein.

\section{COMPARAISONS}

L'aspect fragmentaire ne permet pas de formuler une attribution formelle pour cette dent. Cependant du fait de la présence d'un hypoconide particulièrement crestiforme sur la partie préservée de cette dent et d'un postérolophide développé distalement, cette dent tend à présenter une morphologie proche des taxons basaux Canaanimys et Cachiyacuy. MUSM-3370 differe néanmoins des $\mathrm{m} 3$ de Cachiyacuy contamanensis par sa taille très petite et par la présence d'un postérolophide moins courbé que chez ce dernier; des m3 de Canaanimys maquiensis et de Cachiyacuy kummeli par la présence d'un hypolophide oblique aligné au complexe bras postérieur du protoconide-ectolophide.

Genre et espèce indet. 2

(Fig. 4R, S; Tableau 1)

MATÉRIEL. - MUSM-3381, m2 droite (Fig. 4R); MUSM-3380, dP4 gauche (Fig. 4S).

LoCALITÉ. — TAR-45, Juanjui, Département de San Martín, Pérou. 


\section{DESCRIPTION}

MUSM-3381 et MUSM-3380 sont brachyodontes, tétralophodontes et téniodontes. Elles présentent un contour quadrangulaire. Bien que davantage usée sur la moitié labiale, MUSM-3381 présente quatre cuspides bien marquées (Fig. 4R). Le métaconide est crestiforme et forme l'angle antéro-lingual de la dent. Il présente un bras postérieur très large et long, à l'extrémité duquel se connecte un mésostylide à peine distinct. Le métalophulide I, rectiligne, présente une encoche labiale. Le protoconide, bulbeux, possède un bras postérieur robuste et oblique connecté à une deuxième cristide divisée en deux parties distinctes: une partie labiale peu épaisse d'orientation mésiodistale, et une partie linguale sinueuse et qui s'étend jusqu'à la base du mésostylide, laissant une encoche qui permet la connexion de l'antéroflexide et du mésoflexide. De l'entoconide s'étend un hypolophide transverse et séparé du bras postérieur du protoconide par un fort ectolophide rectiligne, orienté mésiodistalement. L'entoconide présente un fort bras postérieur qui forme un bourrelet, lequel est fusionné au postérolophide. Ce dernier est épais et courbé entre l'entoconide et l'hypoconide, cette cuspide étant crestiforme et oblique. Le bras antérieur issu de l'hypoconide est absent mais l'épaississement basal des cristides peut entraîner une connexion de l'hypoconide avec l'hypolophide aux stades avancés d'usures de la dent.

La dP4 (MUSM-3380; Fig. 4S) présente une forme trapézoïdale due au rapprochement de l'hypocône avec le protocône, plus distal que le paracône. L'antérolophe et le bras antérieur de l'hypocône sont obliques. Ce dernier se connecte à la troisième crête et au protolophe labial, tous deux étant transverses. La connexion entre le protolophe labial et le bras antérieur de l'hypocône est incomplète et forme une encoche. La partie distale de MUSM-3380 est cassée à hauteur du métacône et de l'hypocône.

\section{COMPARAISONS}

Ces deux spécimens, dont la morphologie indique qu'ils appartiennent à des caviomorphes basaux (sensu Boivin et al. 2019a), diffèrent de ceux attribués aux genres Cachiyacuy, Canaanimys et Eoespina par la téniodontie des molaires inférieures; de Cachiyacuy et Canaanimys par la présence d'un protoconide peu crestiforme (à stade d'usure équivalent); d'Eoespina par la présence d'une deuxième cristide transverse incomplète. Il nous paraît à ce jour impossible de les attribuer à un genre ou à une espèce déjà formellement nommés.

\section{MICROSTRUCTURE DE L'ÉMAIL DES INCISIVES}

Les mesures effectuées sur les incisives sont présentées dans leur ensemble en tableau (Tableau 2).

\section{LES TROIS TYPES D'ÉMAIL DÉCUSSANT CHEZ LES RONGEURS}

Chez les mammifères, l'émail est composé de prismes d'hydroxyapatite séparés par des cristallites d'hydroxyapatite formant une matrice inter-prismatique (IPM). La structure de l'émail est souvent divisée en deux catégories: l'émail radial, dans lequel tous les prismes sont orientés dans la même direction, et l'émail décussant, pour lequel on observe des orientations différentes des prismes, formant ainsi une alternance de bandes en vue longitudinale, lesquelles sont nommées bandes d'Hunter-Schreger (HSB). Le schmelzmuster définit l'organisation des différents types d'émail dans l'espace sur une même dent (Koenigswald \& Clemens 1992). Chez les rongeurs et plus précisément sur leurs incisives, ce schmelzmuster se divise en deux portions : la portion externe (PE) qui est formée par l'émail radial et la portion interne (PI) qui comprend les HSB. En fonction de l'inclinaison des HSB et du nombre de prismes qu'elles contiennent, trois types d'émail décussant sont définis: l'émail paucisérié, qui se caractérise par une orientation parallèle à sub-parallèle des cristallites des prismes et de l'IPM, cette dernière étant épaisse et formant une gaine autour des prismes; l'émail unisérié, caractérisé par des HSB monoprismatiques; et enfin l'émail multisérié, dont les HSB sont composées de trois à sept prismes, avec des zones de transition entre les HSB, et une configuration particulière de l'IPM, s'organisant en feuillets fins (Martin 1993). Sur les bases de l'analyse des formes fossiles et de leur position chronologique, l'émail paucisérié est considéré comme la condition primitive chez les rongeurs, à partir de laquelle dérive l'émail unisérié et multisérié (par ex., Martin 1992, 1993).

\section{MicrostruCture DE L'ÉMAIL ET IMPLICATIONS} MACROÉVOLUTIVES CHEZ LES HYSTRICOGNATHI L'émail des incisives chez les rongeurs caviomorphes est un émail multisérié pour lequel Martin (1992, 1993, 1997) a défini trois sous-types fondés principalement sur l'angle formé entre la direction des prismes et celle de l'IPM. Pour le premier, les cristallites des prismes et ceux de l'IPM sont parallèles. Pour le deuxième, l'orientation des cristallites de l'IPM forme un angle aigu avec celle des cristallites des prismes, et l'IPM s'anastomose régulièrement. Enfin, le troisième se caractérise par une orientation des cristallites d'IPM quasi perpendiculaire par rapport à celle des prismes et une absence d'anastomose des feuillets d'IPM. Pour des facilités d'usage, Boivin et al. (2019b) et Marivaux et al. (2019) ont nommé les trois sous-types identifiés par Martin (1992, 1993, 1997) respectivement sous-type 1 , sous-type 2 et sous-type 3 . D'un point de vue biomécanique, ce dernier cas d'agencement est interprété comme un renforcement efficace de l'émail dans les trois dimensions (par ex., Martin 1992, 1993, 1994). Du fait de ces considérations biomécaniques et des occurrences des différents sous-types observés dans le registre fossile, le soustype 1 est considéré comme une condition plésiomorphe de l'émail multisérié. Les sous-types 2 et 3, caractérisés par une augmentation de l'angle formé entre les prismes et les feuillets d'IPM, sont considérés comme des conditions dérivées, le sous-type 3 étant la condition la plus dérivée (Martin 1992, 1993, 1994; pour un résumé, voir Boivin et al. 2019b, Marivaux et al. 2019, et références citées). Chez les caviomorphes, le sous-type 3 n'est observé que chez les octodontoïdes. En outre, des états intermédiaires entre les stades 1-2 et 2-3 ont été observés (Martin 2004, 2005; Vucetich et al. 2010, 2015a; Boivin et al. 2019b). 

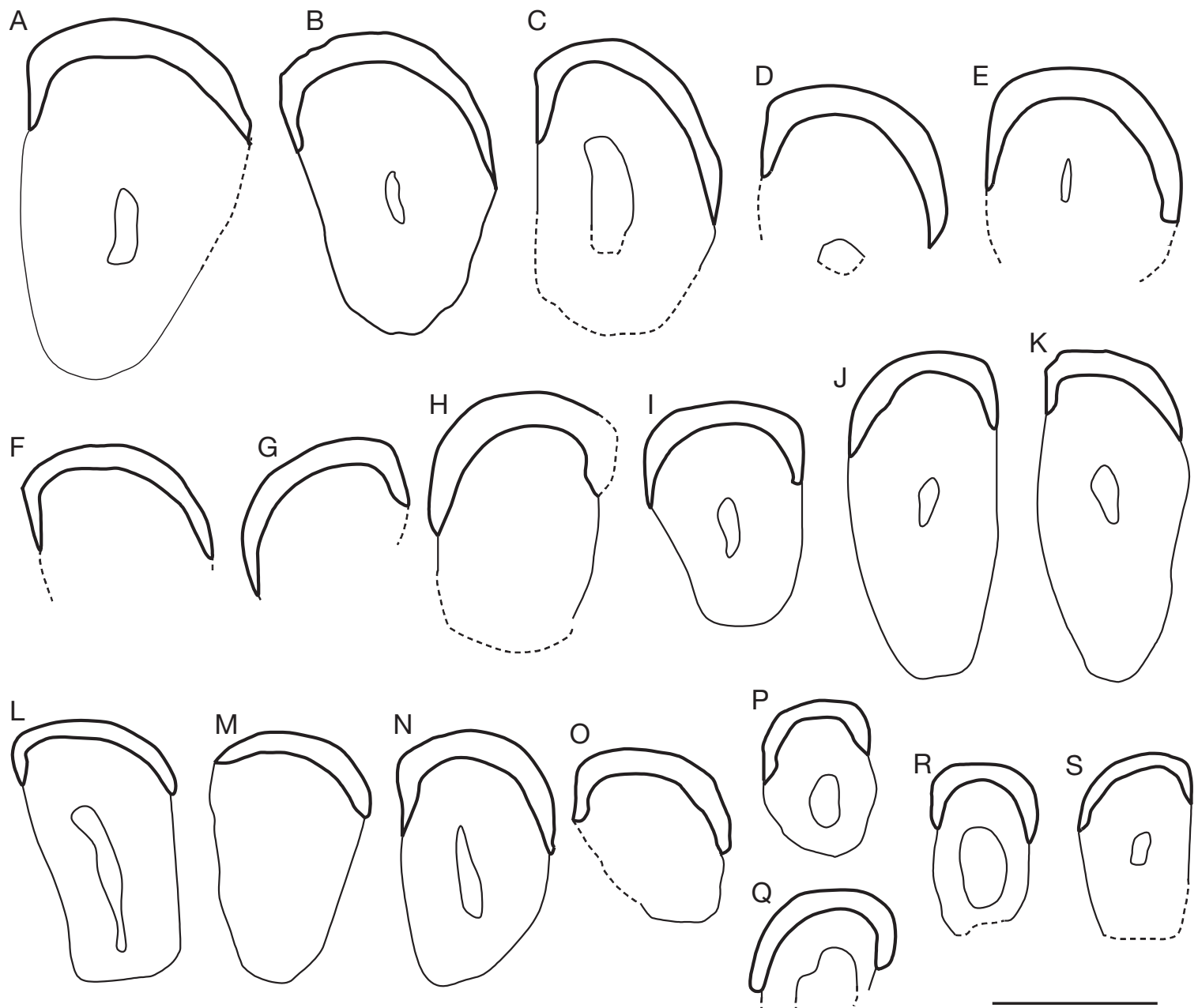

FIG. 5. - Dessins des coupes transversales des incisives des localités de Juanjui et de Balsayacu. A, MUSM-3512 (TAR-55); B, MUSM-3529 (TAR-47); C, MUSM3526 (TAR-47); D, MUSM-3513 (TAR-55); E, MUSM-3528 (TAR-47); F, MUSM-3527 (TAR-47); G, MUSM-3519 (TAR-45); H, MUSM-3530 (TAR-47); I, MUSM-3534 (TAR-49); J, MUSM-3517 (TAR-55bis); K, MUSM-3511 (TAR-55); L, MUSM-3508 (TAR-56); M, MUSM-3509 (TAR-56); N, MUSM-3531 (TAR-47); O, MUSM-3510 (TAR-56); P, MUSM-3539 (TAR-50); Q, MUSM-3533 (TAR-49); R, MUSM-3516 (TAR-55bis); S, MUSM-3518 (TAR-55bis). Échelle: 1 mm.

Pour l'ensemble des localités qui précèdent l'Oligocène, la microstructure de l'émail des incisives de caviomorphes présente un objet d'étude de grand intérêt afin de comprendre l'évolution de cette structure dès les premières phases de diversification du groupe. Récemment, Boivin et al. (2019b) ont effectué une étude des restes d'incisives issus de l'ensemble des localités paléogènes de Contamana (CTA) et de Tarapoto/ Shapaja (TAR). Ces analyses ont montré la présence de soustypes 1, 1-2 et 2 d'émail multisérié dans les localités les plus anciennes d'Amérique du Sud (CTA) et l'apparition des soustypes 2-3 et 3 de manière plus tardive à la transition Éocène/ Oligocène (TAR et Santa Rosa).

\section{MORPHOLOGIE DES INCISIVES}

Faute de pouvoir attribuer formellement des incisives isolés à des taxons, les spécimens analysés ici peuvent être toutefois divisés en trois catégories en fonction de leur taille, et notamment sur la base de leur largeur $(>1 \mathrm{~mm}, 1>\mathrm{x}>0.8 \mathrm{~mm}$ et $<0.8 \mathrm{~mm}$; Fig. 5; Tableau 2). Le diamètre labiolingual n'a pas toujours pu être comparé du fait de l'état fragmentaire de certains spécimens.
MUSM-3512 (Fig. 5A), MUSM-3529 (Fig. 5B), MUSM-3526 (Fig. 5C), MUSM-3513 (Fig. 5D) et MUSM-3528 (Fig. 5E) sont les spécimens représentant la plus grande des trois catégories. Les incisives MUSM-3527 (Fig. 5F), MUSM-3519 (Fig. 5G), MUSM-3530 (Fig. 5H), MUSM-3534 (Fig. 5I), MUSM-3517 (Fig. 5J), MUSM-3511 (Fig. 5K), MUSM-3508 (Fig. 5L), MUSM-3509 (Fig. 5M), MUSM-3510 (Fig. 5N), et MUSM 3531 (Fig. 5O) représentent les incisives de taille intermédiaire, et MUSM-3539 (Fig. 5P), MUSM-3533 (Fig. 5Q), MUSM-3516 (Fig. 5R) et MUSM-3518 (Fig. 5S) représentent les incisives les plus petites de l'assemblage. Cependant la diversité morphologique de ces incisives ne se limite pas à leur taille, et il est possible de distinguer deux morphes parmi les spécimens de la catégorie moyenne: MUSM-3517 (Fig. 5J) et MUSM-3511 (Fig. 5K) présentent une forme plus gracile que les autres spécimens de cette catégorie. Toutefois, ces différences de morphologie pourraient être le reflet de la présence d'incisives supérieures et d'incisives inférieures. En effet, certains des spécimens étudiés ne sont que des minuscules fragments d'incisives de petite taille, et leur section ne permet pas 


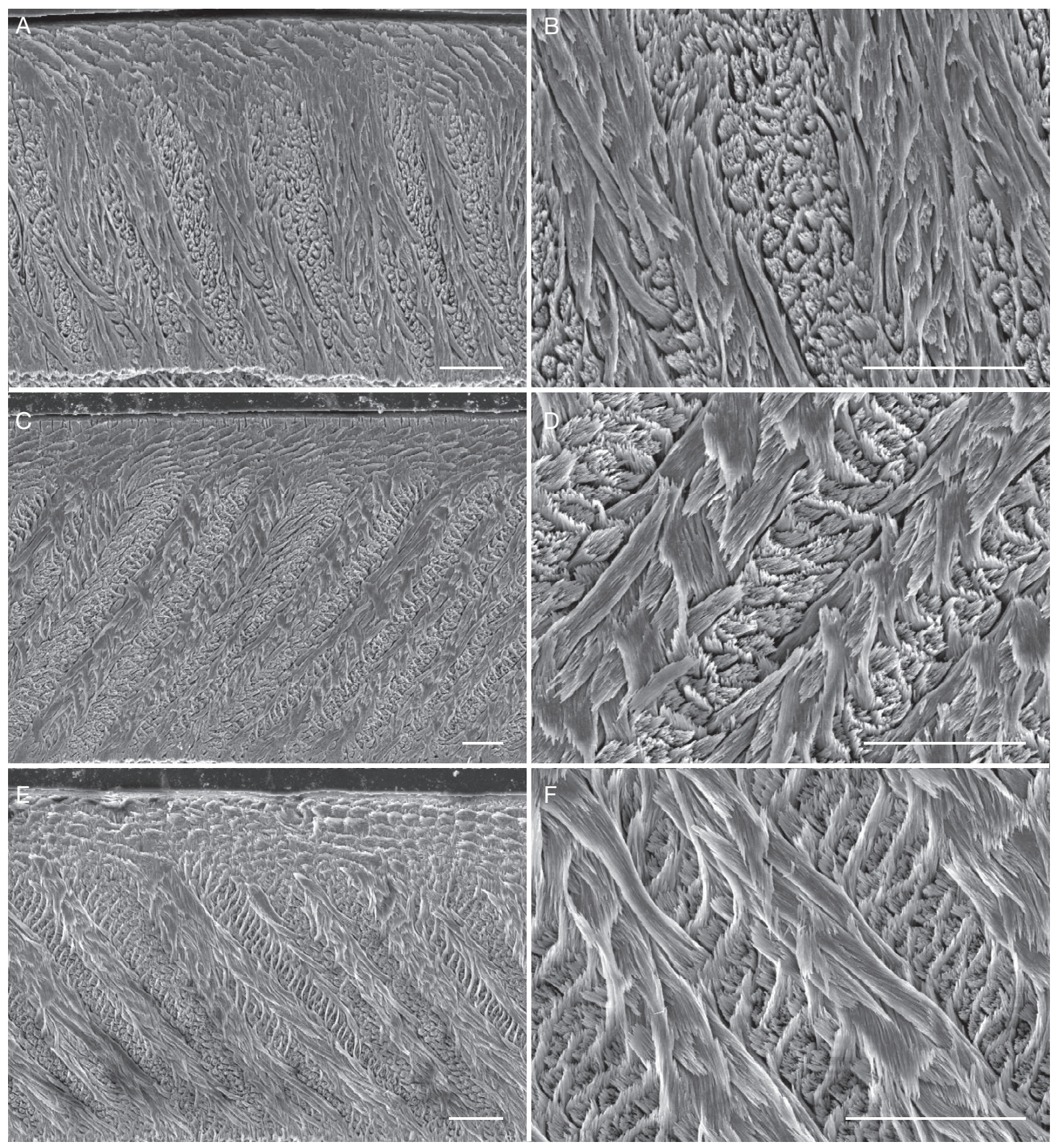

FIG. 6. - Images de microscopie électronique à balayage des trois sous-types d'émail multisérié (en coupe longitudinale) présents à Juanjui/Balsayacu. A, B, MUSM3508 (TAR-56), sous-type 1; C, D, MUSM-3531 (TAR-47), sous-type 2; E, F, MUSM-3534 (TAR-49), sous-type 2-3. Échelle: 20 um.

de juger de leur rayon de courbure, et donc de déterminer s'il s'agit d'incisives inférieures ou supérieures. Parmi la catégorie des plus petites incisives, il est également possible de séparer différents morphes sur la base de la morphologie de la couche d'émail avec d'une part MUSM-3539 (Fig. 5P) et MUSM-3518 (Fig. 5S), et d'autre part MUSM-3533 (Fig. 5Q) et MUSM3516 (Fig. 5R). La comparaison de ce classement par taille et par forme avec la microstructure montre qu'il n'y a pas de lien entre la morphologie des incisives et la microstructure de leur émail dans notre échantillon. Aucune ne présente de sillon dans l'émail, contrairement à ce qui a été décrit sur certaines incisives de Santa Rosa (Martin 2004, 2005).
DESCRIPTION DE LA MICROSTRUCTURE

DE L'ÉMAIL DES INCISIVES

Les différents sous-types de microstructures de l'émail multisérié sont illustrés en Figure 6.

\section{Section de Juanjui (ordre stratigraphique)}

TAR-45. Pour cette localité, la microstructure a été étudiée sur un unique fragment d'incisive (MUSM-3519) présenté en Annexe 1[1,2]. Les deux composantes (PE et PI) de l'émail présentent une épaisseur de $133 \mu \mathrm{m}$ en moyenne dont $18 \%$ de PE. Elle présente un sous-type 1 d'émail multisérié, caractérisé par une angulation faible à inexistante (un angle de $20^{\circ}$ sur la 


\begin{tabular}{|c|c|c|c|c|c|c|c|c|c|c|c|c|}
\hline 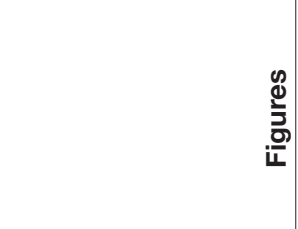 & 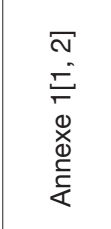 & 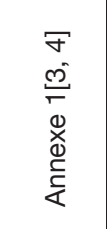 & 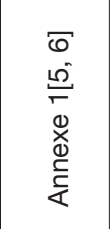 & 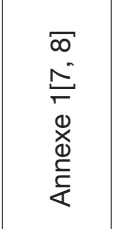 & 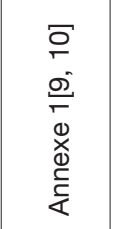 & 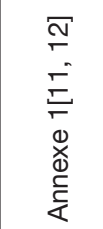 & 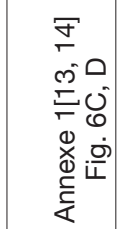 & 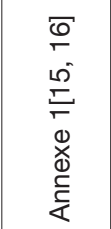 & 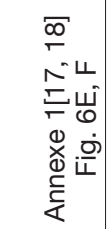 & 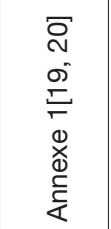 & 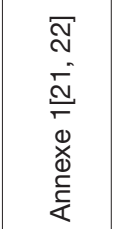 & 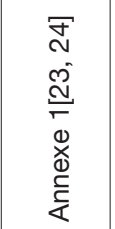 \\
\hline 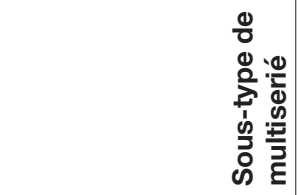 & 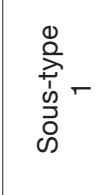 & 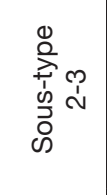 & 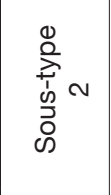 & 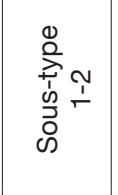 & 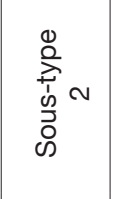 & 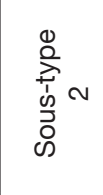 & 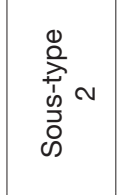 & 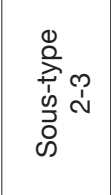 & 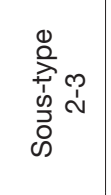 & 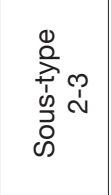 & 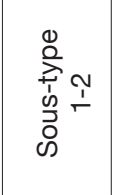 & 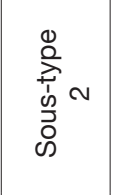 \\
\hline 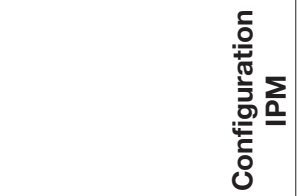 & 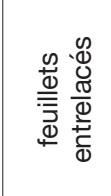 & 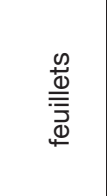 & 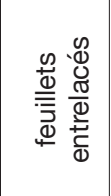 & 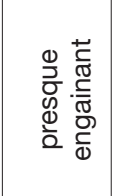 & 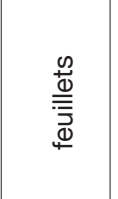 & 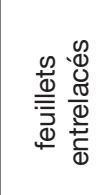 & 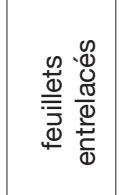 & 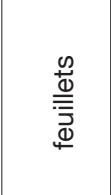 & 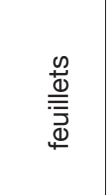 & 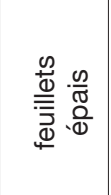 & 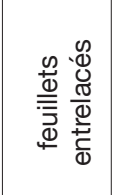 & 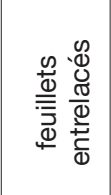 \\
\hline 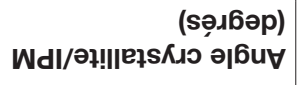 & 尺 10 & $\stackrel{\circ}{\circ} \stackrel{8}{8}$ & ำ & 요 늉 & டூ ட & 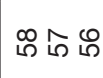 & เిำ 용 & ఫ禺员 & ৪ ட 요 & হNR & เึล ลิ & P श \\
\hline Wdl әsomołseuv & 离 & 离 & 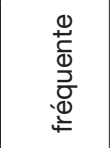 & 童 & 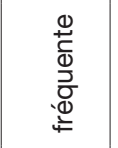 & $\frac{\bar{\pi}}{\frac{\pi}{2}}$ & 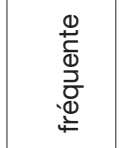 & $\stackrel{\frac{0}{\pi}}{\frac{0}{2}}$ & 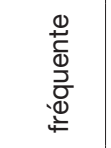 & 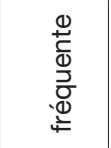 & 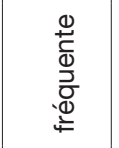 & 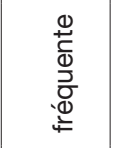 \\
\hline 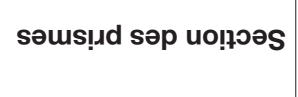 & $\begin{array}{l}\frac{1}{1} \\
\frac{\bar{T}}{0} \frac{\pi}{0} \\
\frac{0}{\alpha}\end{array}$ & $\frac{\overline{\bar{\pi}}}{\frac{\bar{\alpha}}{\sigma}}$ & 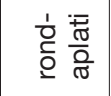 & $\frac{\overline{\bar{T}}}{\frac{\bar{O}}{\alpha}}$ & $\frac{\overline{\bar{\pi}}}{\frac{\bar{\sigma}}{0}}$ & 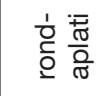 & 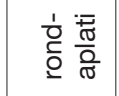 & 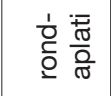 & $\frac{\overline{\bar{\pi}}}{\frac{\bar{\sigma}}{\alpha}}$ & 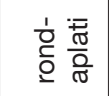 & 옹 & $\frac{\overline{\bar{\pi}}}{\frac{\pi}{0}}$ \\
\hline sgSH səp uo!s!n!a & '̄ & '̄ & '̄ & $\bar{\partial}$ & '̄ & '̄ & '̄ & 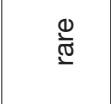 & $\frac{0}{\underline{\underline{N}}}$ & 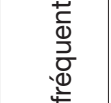 & '̄ & 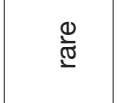 \\
\hline ио!̣!sueג? әр әиоZ & $\frac{\frac{0}{2}}{\frac{0}{50}}$ & $\frac{\frac{0}{2}}{\frac{0}{50}}$ & 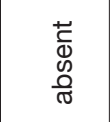 & 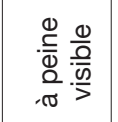 & 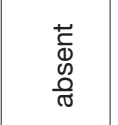 & $\begin{array}{l}\vec{t} \\
\bar{w} \\
0 \\
\sigma \\
\sigma\end{array}$ & $\frac{0}{\frac{0}{0}}$ & 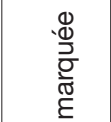 & 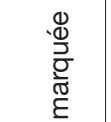 & 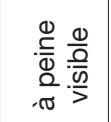 & 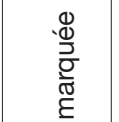 & $\frac{\frac{0}{0}}{\frac{0}{00}}$ \\
\hline gSH גed səus! & ڤें & $\stackrel{+}{\sim}$ & $\stackrel{m}{\sim}$ & હे & $\stackrel{\llcorner}{\sim}$ & $\stackrel{m}{\sim}$ & $\stackrel{m}{\sim}$ & $m$ & $\stackrel{+}{\sim}$ & હे & $\stackrel{\varphi}{+}$ & ले \\
\hline 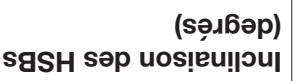 & $\stackrel{\sim}{\sim} \stackrel{\sim}{\sim} \mathbb{N}$ & \& $\stackrel{\mathscr{y}}{\mathcal{y}} \bar{\gamma}$ & mo & $\bar{m} \stackrel{N}{N}$ & Вిల్ల & 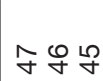 & స్ల & ร์ซำ & 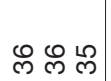 & $\widehat{\sim} \infty \stackrel{\infty}{\infty} \stackrel{\infty}{N}$ & $\stackrel{d}{\sim} \leftarrow 0$ & लॉल \\
\hline 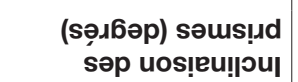 & 6 & م & 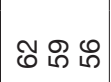 & இசே & $\pi \varnothing \overline{0}$ & ๑ே ஜ & 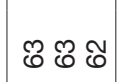 & 呑命员 & No: & 织员 & 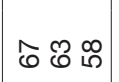 & $\mathscr{\infty}_{\infty}^{\infty} \mathfrak{N}$ \\
\hline Id $\%$ & $\underset{\infty}{\infty}$ & $\stackrel{\infty}{\infty}_{0}^{\infty}$ & $\begin{array}{l}\hat{A} \\
0\end{array}$ & $\overbrace{0}^{N}$ & $\begin{array}{c}\bar{\infty} \\
0 \\
0\end{array}$ & $\begin{array}{l}\infty \\
\infty \\
\infty \\
\infty\end{array}$ & $\cong$ & $\begin{array}{l}n \\
\stackrel{0}{0}\end{array}$ & 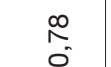 & & $\stackrel{\infty}{\infty}$ & $\underset{\infty}{\infty}$ \\
\hline$\exists d \%$ & $\frac{\infty}{0}$ & ת̃ & $\underset{\substack{N \\
0}}{0}$ & 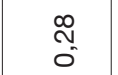 & $\frac{9}{0}$ & $\frac{1}{0}$ & $\frac{\infty}{0}$ & $\stackrel{\sim}{N}$ & 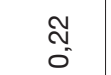 & I & ș & $\frac{\infty}{5}$ \\
\hline 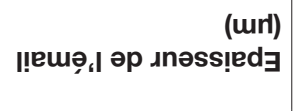 & 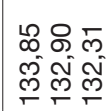 & 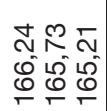 & 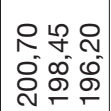 & 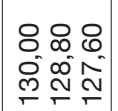 & 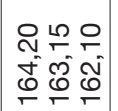 & 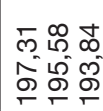 & 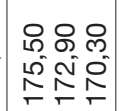 & 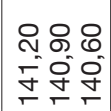 & 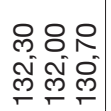 & 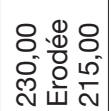 & 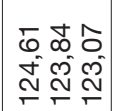 & 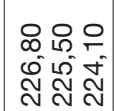 \\
\hline 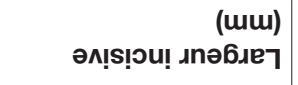 & ô & $\stackrel{0}{-}$ & $\begin{array}{l}\infty \\
\infty \\
\infty\end{array}$ & $\underset{0}{0}$ & o & $\tilde{\sigma}_{-}$ & $\begin{array}{l}\mathscr{8} \\
8 \\
0\end{array}$ & 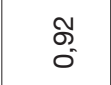 & $\begin{array}{l}\text { 志 } \\
0 \\
0\end{array}$ & $\begin{array}{l}\infty \\
\infty \\
\infty\end{array}$ & $\begin{array}{l}\infty \\
\infty \\
0 \\
0\end{array}$ & $\underset{\sim}{\stackrel{\sim}{\sim}}$ \\
\hline snoo7 & c. & $\stackrel{.}{g}$ & $\stackrel{9}{m}$ & c. & c. & c. & c. & $\stackrel{+}{.}$ & ळ & $\stackrel{+!}{.}$ & क & \\
\hline 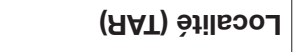 & \& & f & & f & f & f & ร & g & g & 只 & 员 & 员 \\
\hline 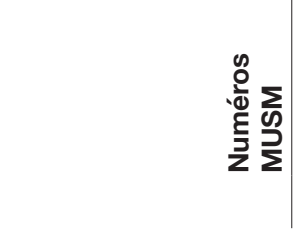 & 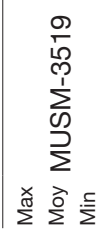 & 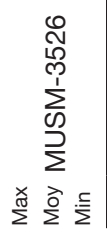 & 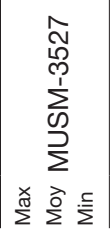 & 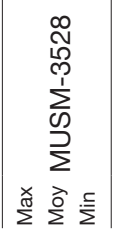 & 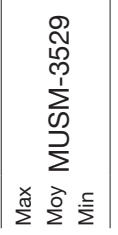 & 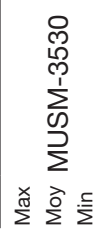 & 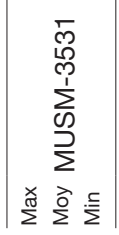 & 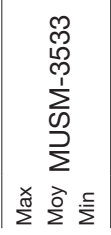 & 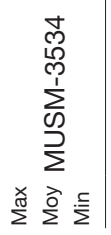 & 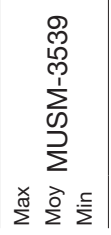 & 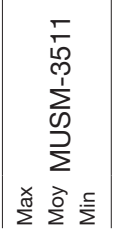 & 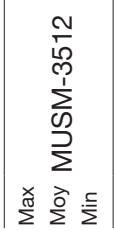 \\
\hline
\end{tabular}




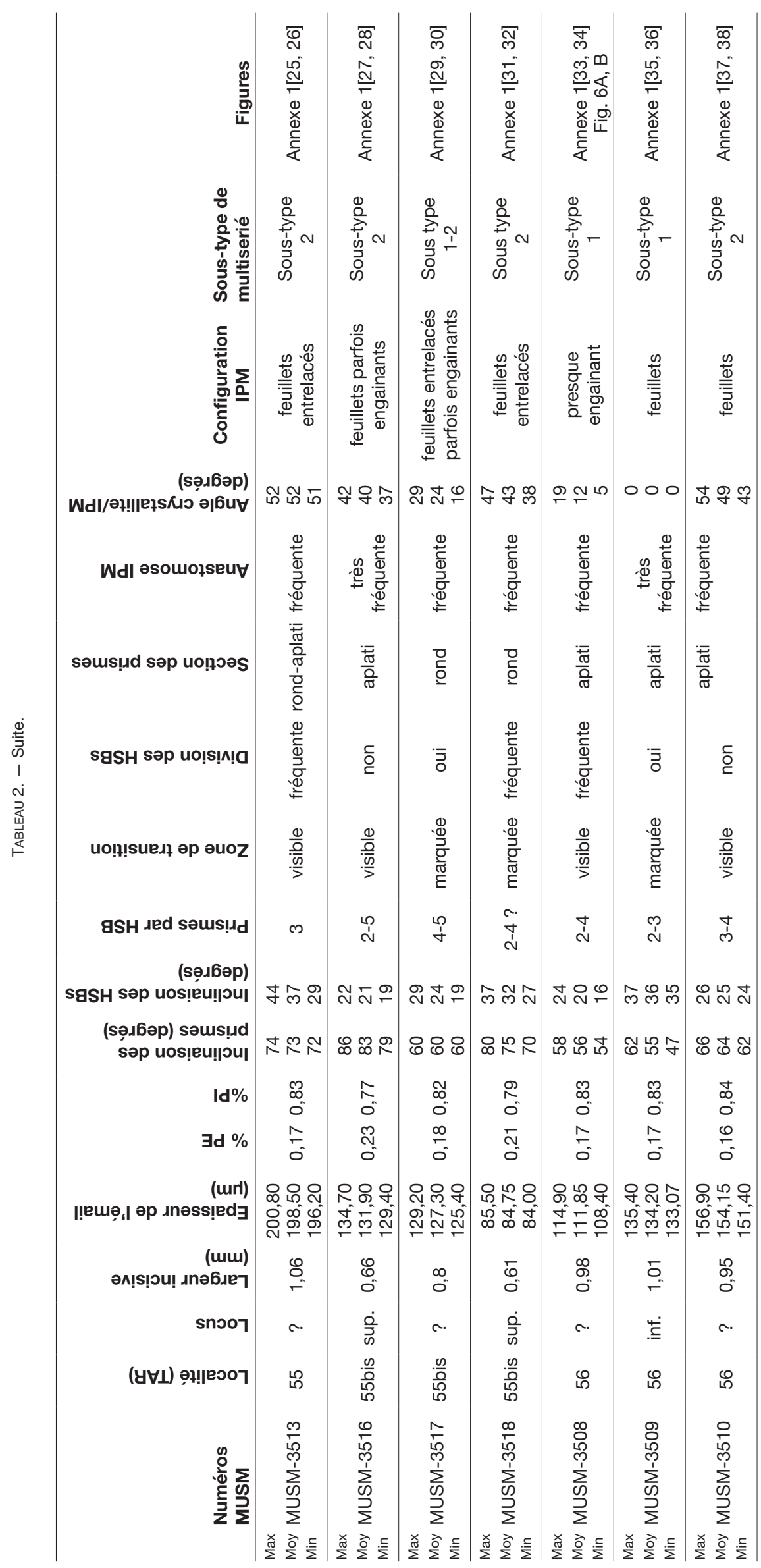


longueur de la coupe) entre les prismes des HSB et les cristallites de l'IPM. Les zones de transition entre HSB sont visibles. Les HSB présentent peu de variabilité dans le nombre de prismes, allant de trois à quatre par bande. En coupe transversale, les prismes sont de section ovale et sont séparés par une IPM en feuillets qui s'anastomosent très fréquemment.

TAR-47. Cette localité, la mieux échantillonnée, présente six fragments d'incisives (MUSM-3526, MUSM-3527, MUSM-3528, MUSM-3529, MUSM-3530 et MUSM-3531; Annexe 1[314]). Les incisives ont des proportions relativement similaires, à l'exception de MUSM-3528 qui, malgré sa largeur d'émail identique aux autres, présente une épaisseur de $c .130 \mu \mathrm{m}$ contre $180 \mu \mathrm{m}$ en moyenne pour les autres incisives. À l'inverse, la PE de cette dernière est plus importante que celle des autres incisives avec $28 \%$ de PE. Cette incisive est également la seule (de cette localité) à présenter un sous-type 1-2 d'émail multisérié, caractérisé par un angle faible (et variable) entre les prismes des HSB et les cristallites de l'IPM, ainsi qu'un arrangement des feuillets d'IPM qui s'anastomosent très fréquemment, ce qui dans une certaine mesure rappelle la structure engainante de l'IPM observée sur des HSB paucisériées. Les prismes sont aplatis et au nombre de trois à quatre par HSB.

Les incisives MUSM-3527, MUSM-3529, MUSM-3530 et MUSM-3531 (Fig. 6C, D) présentent toutes un sous-type 2 d'émail multisérié, montrant une moyenne d'angulation de c. $50^{\circ}$ des cristallites de l'IPM avec les prismes des HSB. Les zones de transition ne sont pas toujours bien définies, bien que souvent visibles. Le nombre de prismes par HSB est variable, allant de 2-3 à 2-5. L'IPM forme des feuillets qui s'anastomosent régulièrement entre les prismes.

La dernière incisive de cette localité est MUSM-3526, laquelle présente un arrangement de l'IPM plus avancé, caractérisant un sous-type 2-3 d'émail multisérié, mais présentant toutefois encore des anastomoses de l'IPM. L'émail mesure en moyenne $165 \mu \mathrm{m}$ d'épaisseur, dont 20\% de PE. Les prismes sont au nombre de deux à quatre par bande, et sont aplatis en section.

TAR-49. Deux fragments d'incisives ont été sélectionnés dans cette localité (MUSM-3533 et MUSM-3534; Annexe 1[15-18]). Ils présentent tous deux un sous-type 2-3 d'émail multisérié, avec un angle prismes HSB/IPM allant de 60 à $65^{\circ}$, et des feuillets d'IPM ne s'anastomosant que très rarement. L'épaisseur de la couche d'émail est très similaire entre les spécimens, avec 140 $\mu \mathrm{m}$ pour MUSM-3533 et $132 \mu \mathrm{m}$ pour MUSM-3534, ainsi qu'un pourcentage de PE de $22 \%$ et $25 \%$, respectivement. Le nombre et la forme des prismes sont variables: MUSM-3533 présente deux à quatre prismes par HSB, dont la section est aplatie, alors que MUSM-3534 (Fig. 6E, F) montre un nombre fixe de trois prismes par bande.

TAR-50. Cette localité n'a livré qu'une seule incisive MUSM3539 (Annexe 1[19, 20]), dont la PE est incomplète et présente des cupules de digestion, comme cela est visible sur l'émail de la molaire MUSM-3535 attribuée à Eoincamys aff. pascuali de la même localité (voir section SYSTÉMATIQUE). Il est donc difficile de mesurer l'épaisseur totale de la couche d'émail, mais il est à noter que celle-ci est importante, avec une PI pouvant aller jusqu'à 200 $\mu \mathrm{m}$. La microstructure décrit un sous-type 2-3 d'émail multisérié. L'angle entre les prismes des HSB et les cristallites de l'IPM est de c. $70^{\circ}$ en moyenne (supérieur à celui observé à TAR-49). L'IPM forme des feuillets épais s'anastomosant régulièrement. Les zones de transition entre HSB sont visibles. Les prismes présentent une section aplatie et sont au nombre de deux à trois par HSB.

\section{Section de Balsayacu (ordre stratigraphique)}

TAR-56. L'étude de la microstructure de l'émail des incisives de cette localité s'est restreinte à trois fragments (MUSM-3508, MUSM-3509 et MUSM-3510; Annexe 1[33-38]), lesquels présentent respectivement une épaisseur totale d'émail (PE + PI) de $134 \mu \mathrm{m}, 112 \mu \mathrm{m}$ et $154 \mu \mathrm{m}$. L'émail des fragments MUSM-3508 (Fig. 6A, B) et MUSM-3509 correspond à un sous-type 1 d'HSB multisériées, alors que MUSM-3510 présente un sous-type 2. Cette détermination repose sur la présence de cristallites d'IPM formant des feuillets dont l'orientation est parallèle à sub-parallèle à celle des prismes $\left(c .0^{\circ}-20^{\circ}\right)$ chez les deux premiers et sur une angulation plus importante $\left(c .49^{\circ}\right)$ chez MUSM-3510. Cependant, MUSM-3508 et MUSM-3509 different par la configuration de l'IPM. En effet, chez MUSM3509, les feuillets d'IPM apparaissent plus fins et s'anastomosent fréquemment, alors que chez MUSM-3508, les feuillets d'IPM s'anastomosent très fréquemment et régulièrement, tendant à former une gaine autour des prismes. Les zones de transition entre HSB sont visibles sur l'émail des trois incisives.

TAR-55 et TAR-55bis. Ces deux localités sont jumelées (deux bancs décimétriques juxtaposés) et ont fourni suffisamment de fragments d'incisives pour qu'il soit possible d'en sélectionner six présentant une couche d'émail non altérée (trois spécimens pour chaque localité: MUSM-3511, MUSM-3512 et MUSM-3513 pourTAR-55, et MUSM-3516, MUSM-3517 et MUSM-3518 pour TAR-55-bis; Annexe 1[21-32]).

À TAR-55, deux des trois incisives (MUSM-3512 et MUSM3513) se caractérisent par une forte épaisseur de l'émail $(225 \mu \mathrm{m}$ et $198 \mu \mathrm{m}$ en moyenne, respectivement) contrairement à MUSM3511 dont l'épaisseur ne dépasse pas $125 \mu \mathrm{m}$. MUSM-3512 et MUSM-3513 ont un sous-type 2 d'HSB multisériées, marquées par des prismes de section aplatie, et formant un angle de c. $50^{\circ}$ avec les cristallites de l'IPM. Les feuillets de cette dernière s'anastomosent très régulièrement.

MUSM-3511 présente un sous-type 1-2 d'HSB multisériées. Les prismes sont au nombre de quatre ou cinq par bande, et ont une section arrondie. Les feuillets d'IPM forment des angles assez faible (c. 20 à $25^{\circ}$ ) avec les prismes, et s'anastomosent très fréquemment. Les zones de transition entre HSB sont bien marquées.

À TAR-55bis, les incisives sont plus petites qu'à TAR-55. MUSM-3516 et MUSM-3517 ont une épaisseur d'émail similaire à MUSM-3511, alors que MUSM-3518 présente un émail très peu épais $(85 \mu \mathrm{m})$. Les pourcentages de PE sont similaires d'une incisive à l'autre (c. 20\%). MUSM-3516 et MUSM-3518 présentent une orientation des feuillets d'IPM déviant de 40 à $45^{\circ}$ par rapport à celle des prismes des HSB, et les prismes ont une section ovale. Ces caractéristiques illustrent un sous-type 2 
TABLEAU 3. - Liste des taxons enregistrés dans les localités paléogènes de Juanjui et de Balsayacu, comparée aux listes des taxons enregistrés à Tarapoto/ Shapaja, Contamana et Santa Rosa (Amazonie péruvienne).

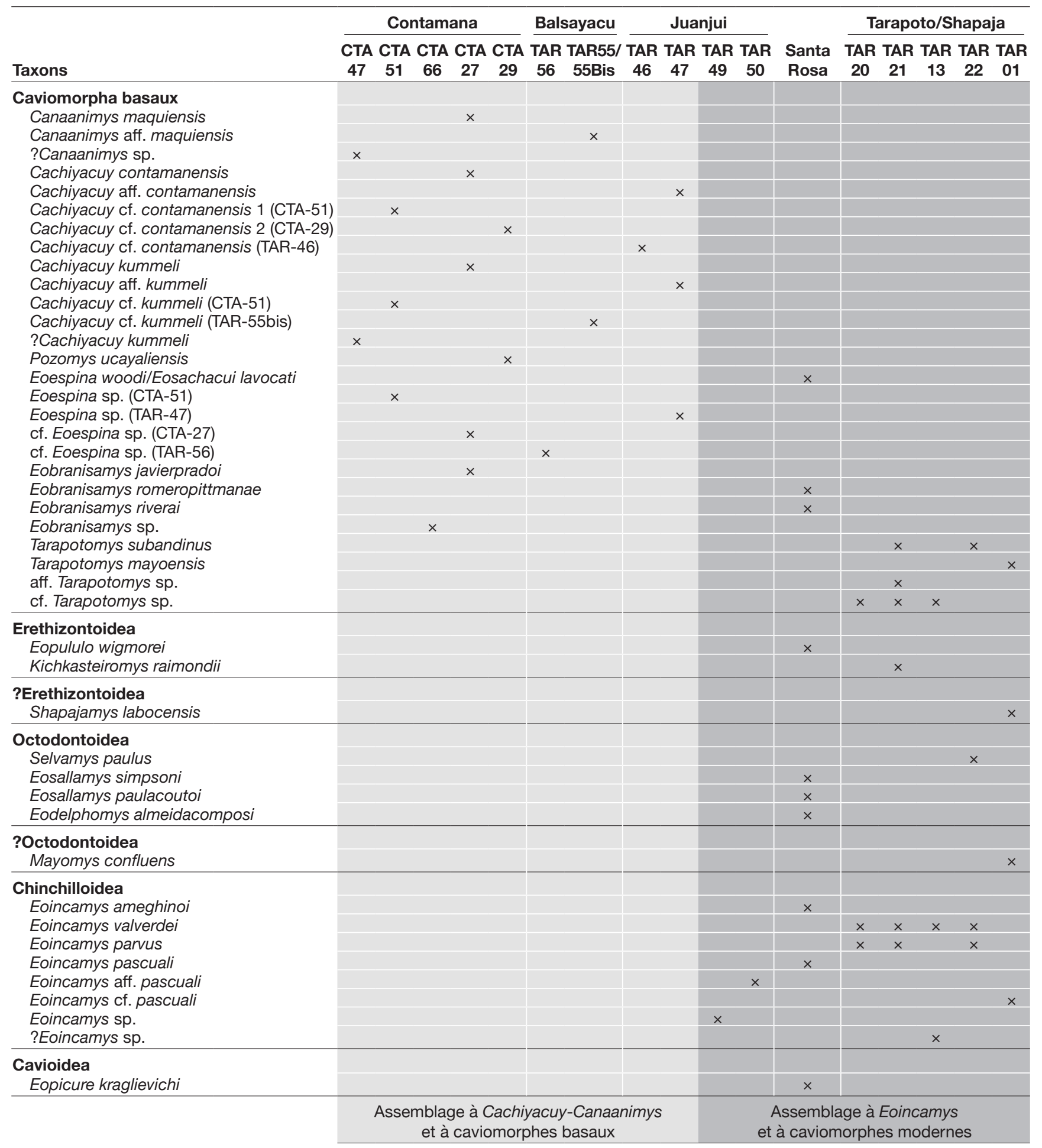

d'émail multisérié. MUSM-3517 présente un émail multisérié caractérisé par des feuillets d'IPM dont l'orientation des cristallites dévie très faiblement de celle des prismes, lesquels ont une section circulaire. Cet arrangement de l'IPM décrit un émail multisérié de sous-type 1-2. Les zones de transitions entre HSB sont beaucoup plus marquées que sur les autres fragments de cette localité. Le nombre de prismes par HSB est très variable pour les incisives de TAR-55-bis: MUSM-3516 présente deux à quatre prismes; MUSM-3517 présente quatre à cinq prismes par bande; et MUSM-3518 présente deux à cinq prismes par bande. Les feuillets d'IPM de l'émail de ces trois incisives s’anastomosent très régulièrement. 


\section{DISCUSSION}

Ces huit nouvelles localités d'Amazonie péruvienne (régions de Juanjui et de Balsayacu), livrent onze taxons de rongeurs, dont sept sont présents dans les localités de TAR-45 à TAR50 , concentrés dans un périmètre restreint et le long d'une série stratigraphique continue (section de Juanjui). Ces espèces illustrent une certaine diversité morphologique, observée tant au niveau des patrons dentaires (structure occlusale) que de la microstructure de l'émail des incisives.

Parmi ces taxons, la majorité des restes dentaires issus des localités TAR-45, TAR-46, TAR-47, TAR-55, TAR-55bis et TAR-56 présentent une affinité avec les genres Canaanimys et Cachiyacuy, tous deux seulement décrits jusqu'alors dans des localités attribuées à la formation Pozo à Contamana, et datées de la fin de l'Éocène moyen (SALMA Barrancien; Antoine et al. 2012; Boivin et al. 2017a). Les localités TAR-49 et TAR-50, situées bien plus haut que TAR-45, TAR-46 et TAR-47 dans la même section (respectivement 490 et 550 mètres au-dessus de TAR-47; Fig. 2) ont en revanche livré des restes dentaires proches du et/ou attribuables au genre Eoincamys, lequel a été défini dans la localité de Santa Rosa (Amazonie péruvienne, Département d'Ucayali; Frailey \& Campbell 2004), puis reconnu dans celles de Tarapoto/Shapaja, à $85 \mathrm{~km}$ de Juanjui (Fig. 1; Tableau 3; Klaus et al. 2017; Boivin et al. 2018). De manière plus anecdotique, les spécimens MUSM-3383 et MUSM-3384, retrouvés dans la localité de TAR-47, ont été attribués à une espèce indéterminée du genre Eoespina (Eoespina sp.). Le spécimen MUSM-3369 de TAR-56 a également été rapproché de ce genre (cf. Eoespina sp.). La pentalophodontie, la brachyodontie et la petite taille des dents, couplées à l'aspect primitif du patron occlusal des dents jugales (finesse et faible obliquité des crêtes et cristides, cuspides encore marquées) chez les taxons rapprochés des genres Cachiyacuy et Canaanimys, tendent à démontrer le cachet primitif de ces formes, qui se situeraient de toute évidence en amont de la divergence et de l'émergence des quatre superfamilles actuelles (Boivin 2017; Boivin et al. 2019a). À l'inverse, la présence dans les localités TAR-49 et TAR-50, de taxons montrant une morphologie plus dérivée (i.e., forte obliquité des lophes ainsi que diminution de leur nombre, associée à une augmentation de la hauteur et taille de la couronne) est un élément en faveur d'un âge nettement plus récent pour ces deux localités de la section de Juanjui. Eoespina, dont la morphologie est peu dérivée (Boivin et al. 2019a), est présent dans la localité de Santa Rosa (Frailey \& Campbell 2004) mais sa première mention remonte à la fin de l'Éocène moyen (CTA-51, Contamana; Boivin et al. 2017a; Tableau 3). Sa présence à TAR-47 n'est donc, à ce titre, pas discriminante. Cela étant, toute affinité des taxons des localités de Juanjui avec ceux issus de localités post-tinguiririciennes d'Amérique du Sud (Oligocène supérieur et au-delà) peut en revanche être écartée du fait du cachet très primitif des restes dentaires et de la faible diversité de patrons occlusaux (voir Boivin et al. 2019a).

L'étude de la microstructure de l'émail sur les incisives issues des localités des sections de Juanjui et de Balsayacu montre la présence d'au moins deux des trois sous-types (1 et 2) d'émail multisérié ainsi que des sous-types intermédiaires 1-2 (TAR-47, TAR-55 et TAR-55bis) et 2-3 (TAR-49 et TAR-50). Sur les deux sections, les localités les plus anciennes stratigraphiquement et livrant les taxons les moins avancés morphologiquement (i.e., TAR-45, TAR-55, TAR-55bis et TAR-56) se sont également avérées être celles dont les incisives de rongeurs présentent une structure de l'émail multisérié la plus primitive, avec notamment une forte fréquence du sous-type 1 (TAR- 45 et TAR-56). En revanche, les localités TAR-49 et TAR-50, localisées au sommet de la série fossilifere de Juanjui et ayant livré des dents jugales de morphologie avancée (Eoincamys), ont révélé des incisives dont l'émail multisérié est de type 2-3, avec notamment une forte angulation entre les prismes des HSB et les cristallites de l'IPM.

Aussi, l'étude des assemblages de rongeurs via différentes méthodes (morphologie et microstructure de l'émail) semblet-elle fournir une indication cohérente sur l'âge relatif des localités à rongeurs des sections de Juanjui et Balsayacu, avec deux ensembles d'âges distincts (TAR-56, TAR-55, TAR-55bis, TAR-45, TAR-46 et TAR-47 d'un côté, et TAR-49 et TAR-50 de l'autre). Ces conclusions sont d'ailleurs corroborées par les éléments fauniques retrouvés en association avec les rongeurs dans les assemblages correspondants: la présence de taxons d'affinités marines, dont le sélacien Pristis (TAR-45 et TAR-47), est une signature des localités de la Formation Pozo, avec un pic d'influence marine pour le membre supérieur de la formation (voir à Tarapoto/Shapaja: Boivin et al. 2018), par contraste avec les formations sous-jacentes et sus-jacentes, essentiellement fluviatiles. Cette influence marine n'est enregistrée ni dans les localités du sommet de la série de Juanjui (TAR-49 et TAR-50), ni dans la série de Balsayacu. TAR-55 a au contraire livré de nombreuses dents du sélacien dulçaquicole Potamotrygon ucayalensis, jusqu'alors restreint au membre inférieur de la Formation Pozo à Contamana (fin de l'Éocène moyen; Adnet et al. 2014; Chabain et al. 2017), et des restes dentaires d'un marsupial prépidolopidé (affinités éocènes; Antoine et al. 2017). Enfin, un marsupial argyrolagoïde aux molaires proto-hypsodontes provenant de TAR-49 est similaire à une forme reconnue à Tarapoto/Shapaja (Oligocène inférieur; Boivin et al. 2018).

En d'autres termes, les localités à rongeurs de Balsayacu (TAR-56, TAR-55 et TAR-55bis) et les gisements les plus bas stratigraphiquement de la section de Juanjui (TAR-45, TAR-46 et TAR-47) appartiennent manifestement au même ensemble, qui peut être attribué avec confiance à l'Éocène. La série de Balsayacu (Fig. 7), livrant Potamotrygon ucayalensis et dépourvue d'influence marine, parait être très proche chronostratigraphiquement du sommet de la séquence fossilifère du membre inférieur de la Formation Pozo à Contamana (CTA-27-CTA-29; fin de l'Éocène moyen). Elle semble également précéder légèrement la séquence TAR-45-TAR-47, qui documente selon toute vraisemblance une fenêtre temporelle inédite de l'histoire évolutive des caviomorphes en Amazonie péruvienne à l'Éocène supérieur (et le retour d'une influence marine évidente; Fig. 7). Tout indique au contraire que TAR49 et TAR-50 enrichissent le registre fossile d'un intervalle stratigraphique nettement postérieur, proche de celui de Santa Rosa et Tarapoto/Shapaja, et attribuable à l'Oligocène inférieur (Fig. 7; Tableau 3). 


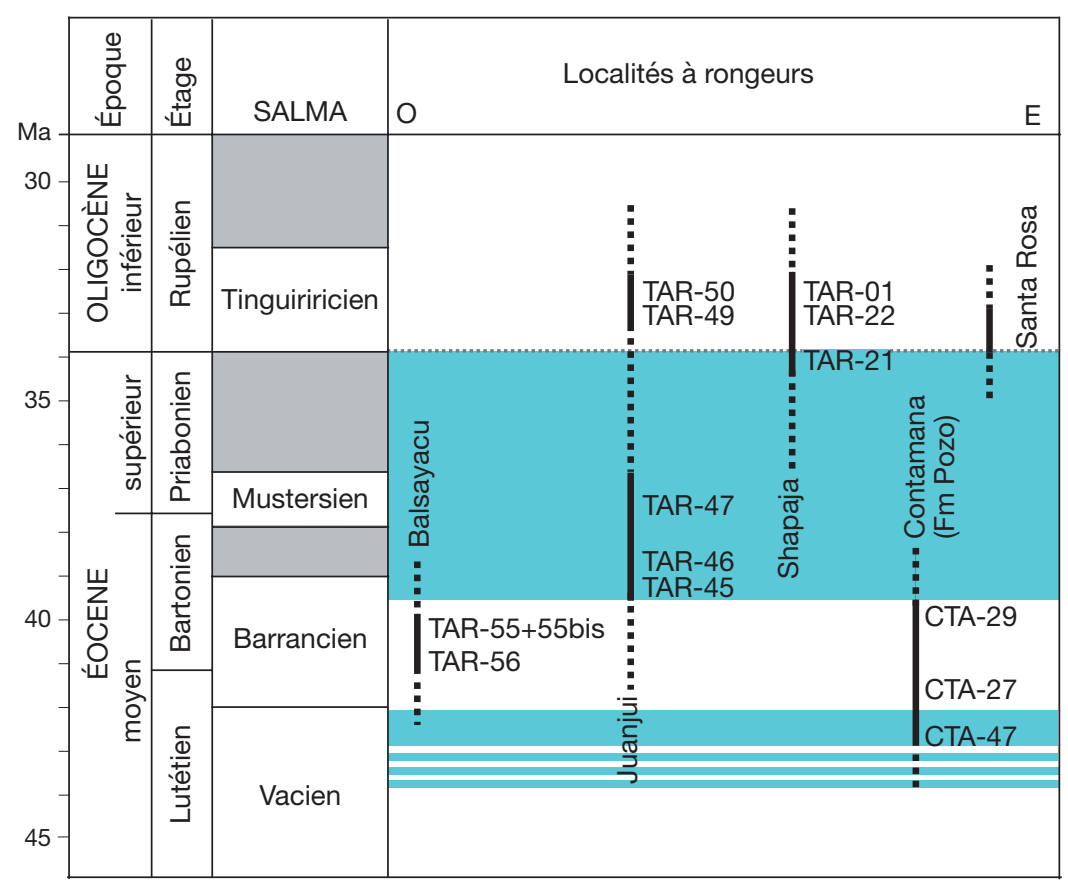

FIG. 7. - Hypothèse d'attribution temporelle des localités de Juanjui et de Balsayacu par rapport aux principales faunes de rongeurs de l'Éocène et de l'Oligocène inférieur d'Amazonie péruvienne. Mis à part pour la section de Contamana, les âges numériques des localités et sections sont hypothétiques. Sur la section de Juanjui, l'écart temporel entre TAR-47 (Priabonien inférieur) et TAR-45/46 (Bartonien) est relatif à l'épaisseur des accumulations sédimentaires. Les fonds bleu et blanc correspondent respectivement à une influence marine et à des environnements purement dulçaquicoles. Modifié et complété d'après Antoine et al. (2016, 2017) et Boivin (2017). Abréviations: E, Est; Fm, Formation; Ma, millions d'années; O, Ouest; SALMA, South American Land Mammal Age.

\section{CONCLUSION}

Cette étude a permis pour la première fois d'identifier des représentants des caviomorphes basaux Cachiyacuy et Canaanimys ailleurs que dans la section fini-éocène moyen de Contamana (Antoine et al. 2012, 2016, 2017; Boivin et al. 2017a), à la fois dans la section de Balsayacu (TAR-55 et TAR-55bis) et à la base de celle de Juanjui (TAR-46 et TAR-47). Ces mentions soutiennent fortement l'âge fini-Éocène moyen à Éocène supérieur des dépôts correspondants. Le genre Eoincamys, décrit originellement à Santa Rosa (Frailey \& Campbell 2004) et récemment reconnu dans des localités de la section de Tarapoto/Shapaja attribuées à l'Oligocène inférieur (Boivin et al. 2018), apparaît en revanche bien plus haut dans la longue section de Juanjui (TAR-49 et TAR-50, respectivement 490 et 560 mètres au-dessus de TAR-47). La parfaite continuité stratigraphique enregistrée dans cette section confirme ainsi l'existence d'un hiatus temporel significatif entre l'intervalle stratigraphique de l'assemblage Canaanimys-Cachiyacuy-Potamotrygon ucayalensis (séquence CTA-47-CTA-29 à Contamana: Boivin et al. 2017a ; TAR-55 à Balsayacu; TAR-46 et TAR-47 à Juanjui) et celui de l'assemblage Eoincamys-Argyrolagoidea (séquence TAR-21-TAR-01 à Tarapoto/Shapaja: Boivin et al. 2018; TAR-49 et TAR-50 à Juanjui). De la même manière et comme avancé par Boivin et al. (2018, 2019b), ce dernier assemblage n'est que très légèrement postérieur à celui de Santa Rosa, qui inclut Eoincamys et des polydolopimorphes, mais seulement non-argyrolagoïdes (Frailey \& Campbell 2004; Goin et al. 2010). Ce long hiatus entre les plus anciennes faunes à rongeurs (séquence CTA-47-CTA-29 à Contamana) et les faunes à Eoincamys était jusqu'alors soutenu sur la seule base de comparaisons fauniques (Antoine et al. 2012, 2016, 2017; Boivin 2017; Boivin et al. 2017a, 2018, 2019b).

Plus généralement, les localités de Juanjui sont également d'un grand intérêt pour l'histoire des écosystèmes néotropicaux, dans la mesure où elles documentent une fenêtre temporelle précédant et entourant la transition Éocène/Oligocène (TEO), peu ou pas documentée jusqu'alors aux basses latitudes d'Amérique du Sud. Cette période est caractérisée par un net refroidissement global (par ex. : Berggren \& Prothero 1992; Zachos et al. 2008), associé à de profondes restructurations des faunes à l'échelle du globe (renouvellements fauniques, extinctions, dispersions; par ex. : Goin et al. 2010). Ainsi, la mise en évidence en Amazonie péruvienne d'une série sédimentaire fossiliferre encadrant la TEO devrait pouvoir permettre de caractériser pour la première fois l'impact éventuel de cet événement sur les faunes et flores des régions néotropicales.

\section{Remerciements}

Nous remercions tous les collègues qui ont participé aux missions de terrain dans la région de Juanjui, Guillaume Billet (Muséum national d'Histoire naturelle [MNHN], Paris), ainsi que toutes les personnes qui nous ont aidés sur le terrain. Un grand merci à Chantal Cazevieille et Alicia Caballero-Megido (Institut des Neurosciences de Montpellier [INM], France) pour l'accès au microscope électronique à balayage (Montpellier RIO Imaging [MRI]). Nous remercions également le Rédacteur en chef, Didier Merle (MNHN) et l'éditeur, Emmanuel Côtez (MNHN), de la revue Geodiversitas, ainsi que Monique 
Vianey-Liaud (ISE-M) et Vincent Lazzari (PALEVOPRIM, Poitiers) pour leur relecture du manuscrit et leurs commentaires qui ont permis son amélioration. Ces missions de terrain ont reçu le soutien financier de la National Geographic Society, de la Leakey Foundation, et ont bénéficié d'une subvention «Investissements d'Avenir" gérée par l'Agence nationale de la Recherche (CEBA, ANR-10-LABX-0025-01). Ces travaux sont publiés dans le cadre de la convention entre le Museo de Historia Natural de Lima/Universidad Nacional Mayor de San Marcos, Lima et l'Institut des Sciences de l'Évolution/ Université de Montpellier. Ils ont également bénéficié de deux programmes de collaborations internationales (FranceArgentine) CoopIntEER CNRS/CONICET et ECOS-SUD/ FONCyT. Publication ISE-M 2019-116-Sud.

\section{RÉFÉRENCES,}

Adnet S., Salas Gismondi R. \& Antoine P.-O. 2014. - Comparisons of dental morphology in river stingrays (Chondrichthyes: Potamotrygonidae) with new fossils from the middle Eocene of Peruvian Amazonia rekindle debate on their evolution. Naturwissenschaften 101: 33-45. https://doi.org/10.1007/s00114-013-1127-1

Antoine P.-O., Marivaux L., Croft D. A., Billet G., Ganerød M., Jaramillo C., Martin T., Orliac M. J., Tejada J., Altamirano A. J., Duranthon F., Fanjat G., Rousse S. \& Salas GISMONDI R. 2012. - Middle Eocene rodents from Peruvian Amazonia reveal the pattern and timing of caviomorph origins and biogeography. Proceedings of the Royal Society of London, Biological Sciences 279: 1319-1326. https://doi.org/10.1098/ rspb.2011.1732

Antoine P.-O., Abello M. A., Adnet S., Altamirano Sierra A. J., Baby P., Billet G., Boivin M., Calderón Y., Candela A., Chabain J., Corfu F., Croft D. A., Ganerød M., Jaramillo C., Klaus S., Marivaux L., Navarrete R. E., Orliac M. J., Parra F., Pérez M. E., Pujos F., Rage J.-C., Ravel A., Robinet C., Roddaz M., Tejada-Lara J. V., Vélez-Juarbe J., Wesselingh F. P. \& SALAS-GISMONDI R. 2016. — A 60-million-year Cenozoic history of western Amazonian ecosystems in Contamana, eastern Peru. Gondwana Research 31: 30-59. https://doi.org/10.1016/j. gr.2015.11.001

Antoine P.-O., Salas-Gismondi R., Pujos F., Ganerød M. \& MARIVAUX L. 2017. - Western Amazonia as a hotspot of mammalian biodiversity throughout the Cenozoic. Journal of Mammalian Evolution 24: 5-17. https://doi.org/10.1007/s10914-016-9333-1

BARBIÈRE F. \& MARIVAUX L. 2015. - Phylogeny and evolutionary history of hystricognathous rodents from the Old World during the Tertiary: new insights into the emergence of modern "phiomorph" families, in COX P. G. \& HAUTIER L. (eds), Evolution of the Rodents - Advances in Phylogenetics, Functional Morphology and Development. Cambridge University Press, Cambridge: 97-120.

BergGren W. A. \& Prothero D. R. (eds) 1992. —Eocene-Oligocene Climatic and Biotic Evolution: an Overview. Princeton University Press, Princeton, 568 p.

Bertrand O. C., Flynn J. J., Croft D. A. \& Wyss A. R. 2012. Two New Taxa (Caviomorpha, Rodentia) from the Early Oligocene Tinguiririca fauna (Chile). American Museum Novitates 3750: 1-36. https://doi.org/10.1206/3750.2

Borvin M. 2017. - Rongeurs paléogènes d'Amazonie péruvienne: anatomie, systématique, phylogénie et paléobiogéographie. Volume de Thèse, Université de Montpellier, France, 608 p.

BOIVIN M. \& MARIVAUX L. 2018. — Dental homologies and evolutionary transformations in Caviomorpha (Hystricognathi, Rodentia): new data from the Paleogene of Peruvian Amazonia. Historical Biology. https://doi.org/10.1080/08912963.2018.1506778
Boivin M., Marivaux L., Orliac M. J., Pujos F., Salas-GisMONDi R., Tejada-Lara J. V. \& Antoine P.-O. 2017a. — Late middle Eocene caviomorph rodents from Contamana, Peruvian Amazonia. Paleontologia Electronica 20 (1.19A): 1-50. https:// doi.org/10.26879/742

Boivin M., Marivaux L., Candela A. M., Orliac M. J., Pujos F., Salas-Gismondi R., Tejada-Lara J. V. \& Antoine P.-O. 2017b. Late Oligocene caviomorph rodents from Contamana, Peruvian Amazonia. Papers in Palaeontology 3: 69-109. https://doi. org/10.1002/spp2.1068

Boivin M., Marivaux L., Pujos F., Salas-Gismondi R., TejadaLara J., Varas-Malca R. \& Antoine P.-O. 2018. — Early Oligocene caviomorph rodents from Shapaja, Peruvian Amazonia. Palaeontographica Abteilung A, 311: 87-156. https://doi. org $/ 10.1127 / \mathrm{pala} / 2018 / 0075$

Boivin M., Marivaux L. \& Antoine P.-O. 2019a. — L'apport du registre paléogène d'Amazonie sur la diversification initiale des Caviomorpha (Hystricognathi, Rodentia): implications phylogénétiques, macroévolutives et paléobiogéographiques. Geodiversitas 41 (4): 143-245. https://doi.org/10.5252/geodiversitas2019v41a4. http://geodiversitas.com/41/4

Boivin M., Marivaux L., Pujos F., Salas-Gismondi R., Vieytes E. \& ANTOINE P.-O. 2019b. - Incisor enamel microstructure of Palaeogene caviomorph rodents from Contamana and Shapaja (Peru). Journal of Mammalian Evolution 26 (3): 389-406. https:// doi.org/10.1007/s10914-018-9430-4

Candela A. M., Rasia L. L. \& Pérez M. E. 2012. - Early Miocene paleobiology in Patagonia: paleobiology of Santacrucian caviomorph rodents: a morphofunctional approach, in VIZCAínO S. F., KAY R. F. \& BARGO M. S. (eds), Early Miocene Paleobiology in Patagonia: High-Latitude Paleocommunities of the Santa Cruz Formation. Cambridge University Press, New York: 287-305. https:// doi.org/10.1017/CBO9780511667381.016

Chabain J., Antoine P.-O., Altamirano-Sierra A. J., Marivaux L., Pujos F., Salas-Gismondi R \& Adnet S. 2017. - Cenozoic batoid record from Contamana, Peru, with special focus on freshwater potamotrygonines (Chondrichthyes, Myliobatiformes) from the Pebas wetland system. Geobios 50: 389-400. https://doi. org/10.1016/j.geobios.2017.10.003

Eude A., Roddaz M., Brichau S., Brusset S., Calderón Y., BABY P. \& Soula J. C. 2015. - Controls on timing of exhumation and deformation in the northern Peruvian eastern Andean wedge as inferred from low-temperature thermochronology and balanced cross section. Tectonics 34: 715-730. https://doi. org/10.1002/2014TC003641

Fabre P.-H., GAlewski T., Tilak M. \& Douzery E. 2013. — Diversification of South American spiny rats (Echimyidae): a multigene phylogenetic approach. Zoologica Scripta 42: 117-134. https:// doi.org/10.1111/j.1463-6409.2012.00572.x

Fabre P.-H., Hautier L. \& Douzery E. 2015. - A synopsis of rodent molecular phylogenetics, systematics and biogeography, in COX P. G. \& HAUTIER L. (eds), Evolution of the Rodents - Advances in Phylogenetics, Functional Morphology and Development. Cambridge University Press, Cambridge: 19-69.

FIELDS R. W. 1958. - Hystricomorph rodents from the late Miocene of Colombia, South America. Geologiska Föreningen i Stockholm Förhandlingar 80: 246-246. https://doi.org/10.1080/11035895809447240

Frailey C. D. \& CAMpbeLl K. E. 2004. - Paleogene rodents from Amazonian Peru: the Santa Rosa local fauna, in CAMPBELL K. E. (ed.), The Paleogene Mammalian Fauna of Santa Rosa, Amazonian Peru. Natural History Museum of Los Angeles County, Los Angeles: 71-130.

Goin F. J., Abello M. A. \& Chornogubsky L. 2010. — Middle Tertiary marsupials from central Patagonia (early Oligocene of Gran Barranca): understanding South America's Grande Coupure, in Madden R. H., Carlini A. A., Vucetich M. G. \& KaY R. F. (eds), The Paleontology of Gran Barranca. Cambridge University Press, Cambridge: 69-105. 
Hermoza W., Brusset S., Baby P., Gil W., Roddaz M., GuerRERO N. \& BolaÑos R. 2005. - The Huallaga foreland basin evolution: Thrust propagation in a deltaic environment, northern Peruvian Andes. Journal of South American Earth Sciences 19: 21-34. https://doi.org/10.1016/j.jsames.2004.06.005

Hurtado C., Roddaz M., Ventura Santos R., Baby P., Antoine P.-O. \& Dantas E. 2018. — Late Cretaceous-early Paleocene drainage shift of Amazonian rivers driven by Equatorial Atlantic Ocean opening and Andean uplift as deduced from the provenance of northern Peruvian sedimentary rocks (Huallaga basin). Gondwana Research 63: 152-168. https://doi. org/10.1016/j.gr.2018.05.012

Klaus S., MagalHaes C., Salas-Gismondi R., Gross M. \& Antoine P.-O. 2017. - Paleogene and Neogene brachyurans of the Amazon basin: a revised first appearance date for primary freshwater crabs (Crustacea, Brachyura, Trichodactylidae). Crustaceana 90: 953-967. https://doi.org/10.1163/15685403-00003629

KOENIGSWALD W. V. \& CLEMENS W. A. 1992. — Levels of complexity in the microstructure of mammalian enamel and their application in studies of systematics. Scanning Microscopy 6: 195-218.

KoEnigsWald W. V. \& SANDER P. M. 1997. — Glossary of terms used for enamel microstructures, in KOENIGSWALD W. V. \& SANDER P. M. (eds), Tooth Enamel Microstructure. Balkema, Rotterdam: 267-280.

LAVOCAT R. 1976. - Rongeurs caviomorphes de l'Oligocène de Bolivie. II. Rongeurs du bassin déséadien de Salla-Luribay (collection Hoffstetter). Palaeovertebrata 7 (3): 15-90.

Mares M. A. \& OJedA H. H. 1982. - Patterns of diversity and adaptation in south amercian hystricognath rodents, in Mares M. A. \& Genoways H. H. (eds), Mammalian Biology in South America.Pittsburgh University, Pittsburgh: 393-433.

Marivaux L., Vianey-Liaud M. \& Jaeger J.-J. 2004. - Highlevel phylogeny of early Tertiary rodents: dental evidence. Zoological Journal of the Linnean Society 142: 105-134. https://doi. org/10.1111/j.1096-3642.2004.00131.x

Marivaux L., Essid E. M., Marzougui W., Khayati Ammar H., Adnet S., Marandat B., Merzeraud G., Tabuce R \& VianeyLIAUD M. 2014. - A new and primitive species of Protophiomys (Rodentia, Hystricognathi) from the late middle Eocene of Djebel el Kébar, Central Tunisia. Paleovertebrata 38 (1): 1-17. http:// dx.doi.org/10.18563/pv.38.1.e2

Marivaux L., Boivin M., Adnet S., Benammi M., Tabuce R. \& BENAMMI M. 2019. - Incisor enamel microstructure of hystricognathous and anomaluroid rodents from the earliest Oligocene of Dakhla, Atlantic Sahara (Morocco). Journal of Mammalian Evolution 26 (3): 373-388. https://doi.org/10.1007/s10914-017-9426-5

MARTIN T. 1992. - Schmelzmikrostruktur in den Inzisiven altund neuweltlicher hystricognather Nagetiere. Palaeovertebrata mémoire extraordinaire: 1-168.

MARTIN T. 1993. — Early rodent incisor enamel evolution: phylogenetic implications. Journal of Mammalian Evolution 1: 227-254. https://doi.org/10.1007/BF01041665

MARTIN T. 1994. - On the systematic position of Chaetomys subspinosus (Rodentia: Caviomorpha) based on evidence from the incisor enamel microstructure. Journal of Mammalian Evolution 2: 117-131. https://doi.org/10.1007/BF01464364

MARTIN T. 1997. - Incisor enamel microstructure and systematics in rodents, in Koenigswald W. V. \& SANDer P. M. (eds), Tooth Enamel Microstructure. Balkema, Rotterdam: 163-175.

MARTIN T. 2004. - Incisor enamel microstructure of South America's earliest rodents: implications for caviomorph origin and diversification, in CAMPBELL K. E. (ed), The Paleogene Mammalian Fauna of Santa Rosa, Amazonian Peru. Natural History Museum of Los Angeles County, Los Angeles: 131-140.

MARTIN T. 2005. - Incisor schmelzmuster diversity in South America's oldest rodent fauna and early caviomorph history. Journal of Mammalian Evolution 12: 405-417. https://doi.org/10.1007/ s10914-005-6968-8

Patton J. L., Pardiñas U. F. J. \& Elía G. D’ 2015. — Mammals of South America. Volume 2. Rodents. University of Chicago Press, Chicago, $1384 \mathrm{p}$.

Roddaz M., Hermoza W., Mora A., Baby P., Parra M., ChristopHOUL F., BRUSSET S. \& ESPURT N. 2010. — Cenozoic sedimentary evolution of the Amazonian foreland basin system, in HOORN C. \& WeSSELINGH F. P. (eds), Amazonia, Landscape and Species Evolution: a Look into the Past. Blackwell-Wiley, Hoboken, 61-88.

SÁnCHEZ FernándeZ A. W. \& HerrerA TuFINO I. 1998. — Geología de los cuadrángulos de Moyobamba, Saposoa y Juanjui 13-j, 14-j y 15-j. [Boletín A-122]. Instituto Geológico, Minero y Metalúrgico - INGEMMET.

Tabuce R., Delmer C. \& GHeErbrant E. 2007. - Evolution of the tooth enamel microstructure in the earliest proboscideans (Mammalia). Zoological Journal of the Linnean Society 149: 611628. https://doi.org/10.1111/j.1096-3642.2007.00272.x

Vélez-Juarbe J., Martin T., MacPhee R. D. E. \& Ortega-Ariza D. 2014. - The earliest Caribbean rodents: Oligocene caviomorphs from Puerto Rico. Journal of Vertebrate Paleontology 34: 157-163. https://doi.org/10.1080/02724634.2013.789039

Vucetich M. G., Vieytes E. C., Perez M. E. \& Carlini A. A. 2010. The rodents from La Cantera and the early evolution of caviomorphs in South America, in Madden R. H., Carlini A. A., Vucetich M. G. \& KaY R. F. (eds), The Paleontology of Gran Barranca. Cambridge University Press, Cambridge: 193-205.

Vucetich M. G., Arnal M., Deschamps C. M., Pérez M. E. \& VIEYTES E. C. 2015A. - A brief history of caviomorph rodents as told by the fossil record, in VASSALLO A. I. \& ANTENUCCI D. (eds), Biology of Caviomorph Rodents: Diversity and Evolution. Sociedad Argentina para el Estudio de los Mamíferos (SAREM), Buenos Aires, Argentina: 11-62.

Vucetich M. G., Dozo M. T., Arnal M. \& Pérez M. E. 2015b. New rodents (Mammalia) from the Late Oligocene of Cabeza Blanca (Chubut) and the first rodent radiation in Patagonia. Historical Biology 27: 236-257. https://doi.org/10.1080/08912 963.2014 .883506

Wilson D. E. \& ReEder D. M. 2005. - Mammal Species of the World: A Taxonomic and Geographic Reference. Johns Hopkins University Press, 2142 p.

Wood A. E. 1949. - A new Oligocene rodent genus from Patagonia. American Museum Novitates 1435: 1-54. http://hdl.handle. net $/ 2246 / 2350$

WoOd A. E. \& WiLSON R. W. 1936. — A suggested nomenclature for the cusps of the cheek teeth of rodents. Journal of Paleontology 10: 388-391. https://www.jstor.org/stable/1298483

WoOd A. E. \& PATterson B. 1959. - The rodents of the Deseadan Oligocene of Patagonia and the beginnings of South American rodent evolution. Bulletin of the Museum of Comparative Zoology 120: 281-428. https://biodiversitylibrary.org/page/4780786

Wyss A. R., Flynn J. J., Norell M. A., Swisher C. C., Charrier R., NovaceK M. J. \& McKenna M. C. 1993. - South America's earliest rodent and recognition of a new interval of mammalian evolution. Nature 365: 434-437. https://doi.org/10.1038/365434a0

Zachos J. C., Dickens G. R. \& Zeebe R. E. 2008 - An early Cenozoic perspective on greenhouse warming and carbon-cycle dynamics. Nature 451: 279-283. https://doi.org/10.1038/nature06588 
ANNEXE 1. - Images de microscopie à balayage de la structure de l'émail multisérié en coupe longitudinale chez les rongeurs de la région de Juanjui. Détail en fin d'Annexe.
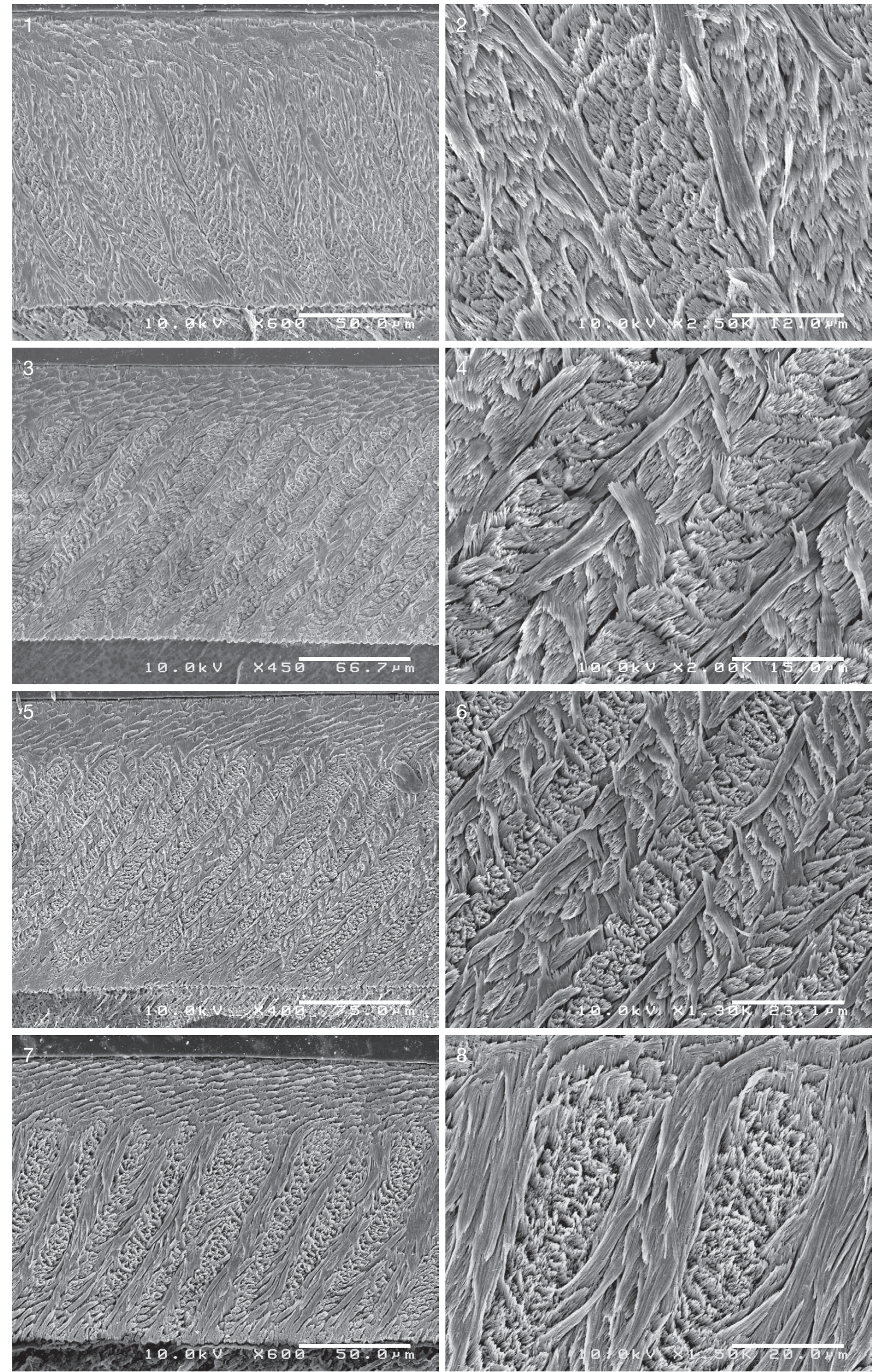
AnNEXe 1. - Suite.
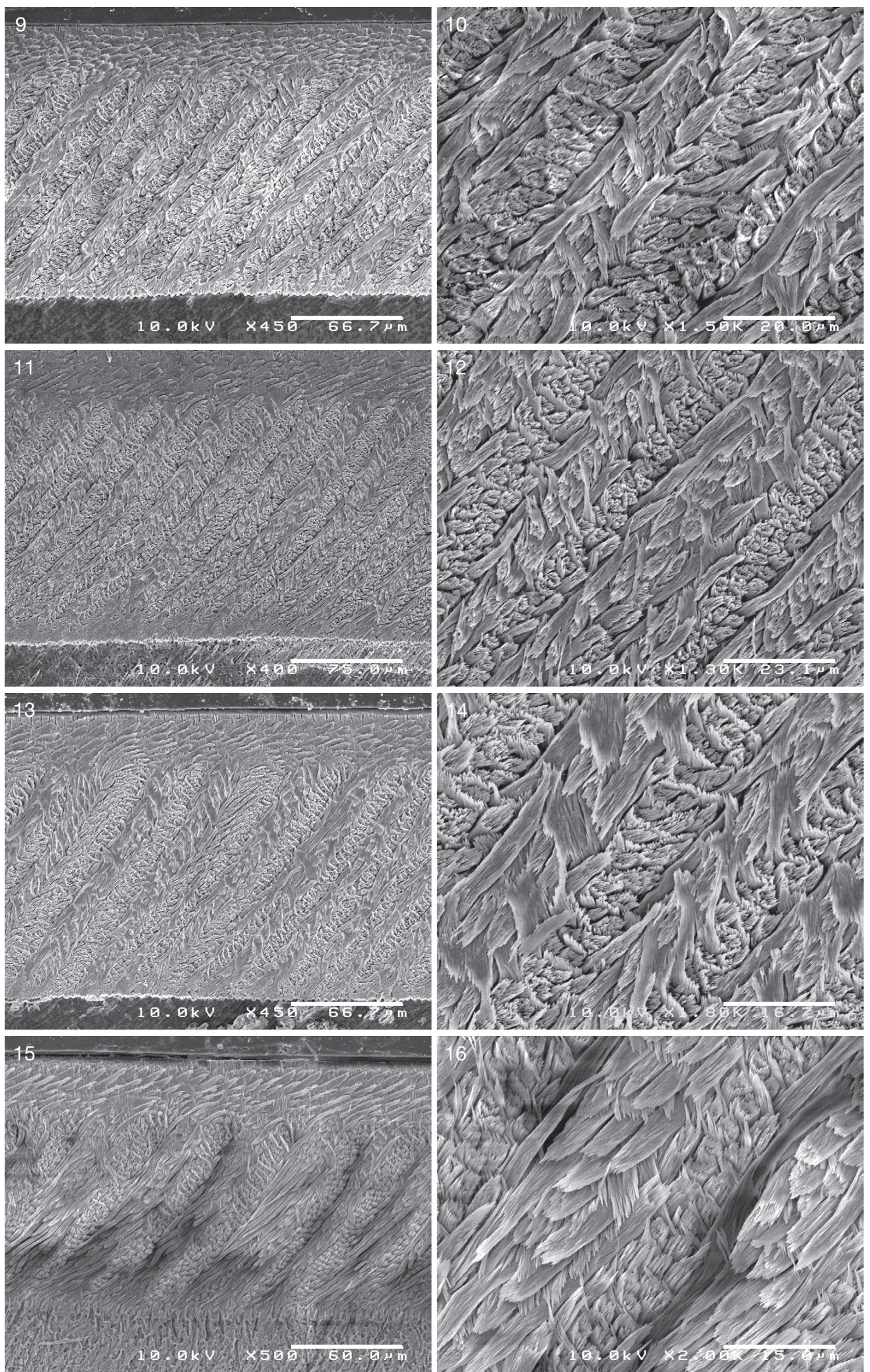
ANNEXE 1. - Suite.
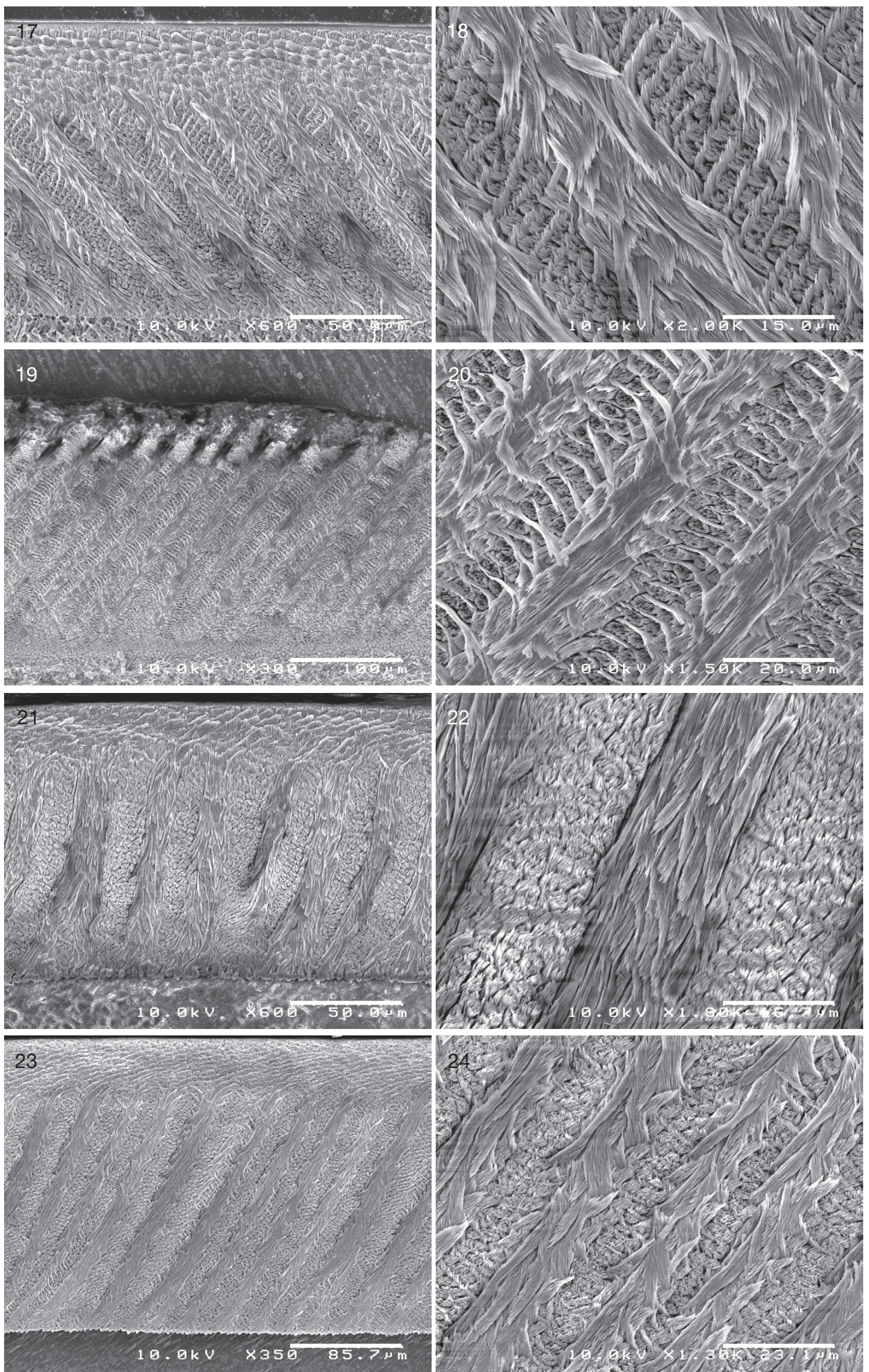
ANNEXE 1. - Suite.

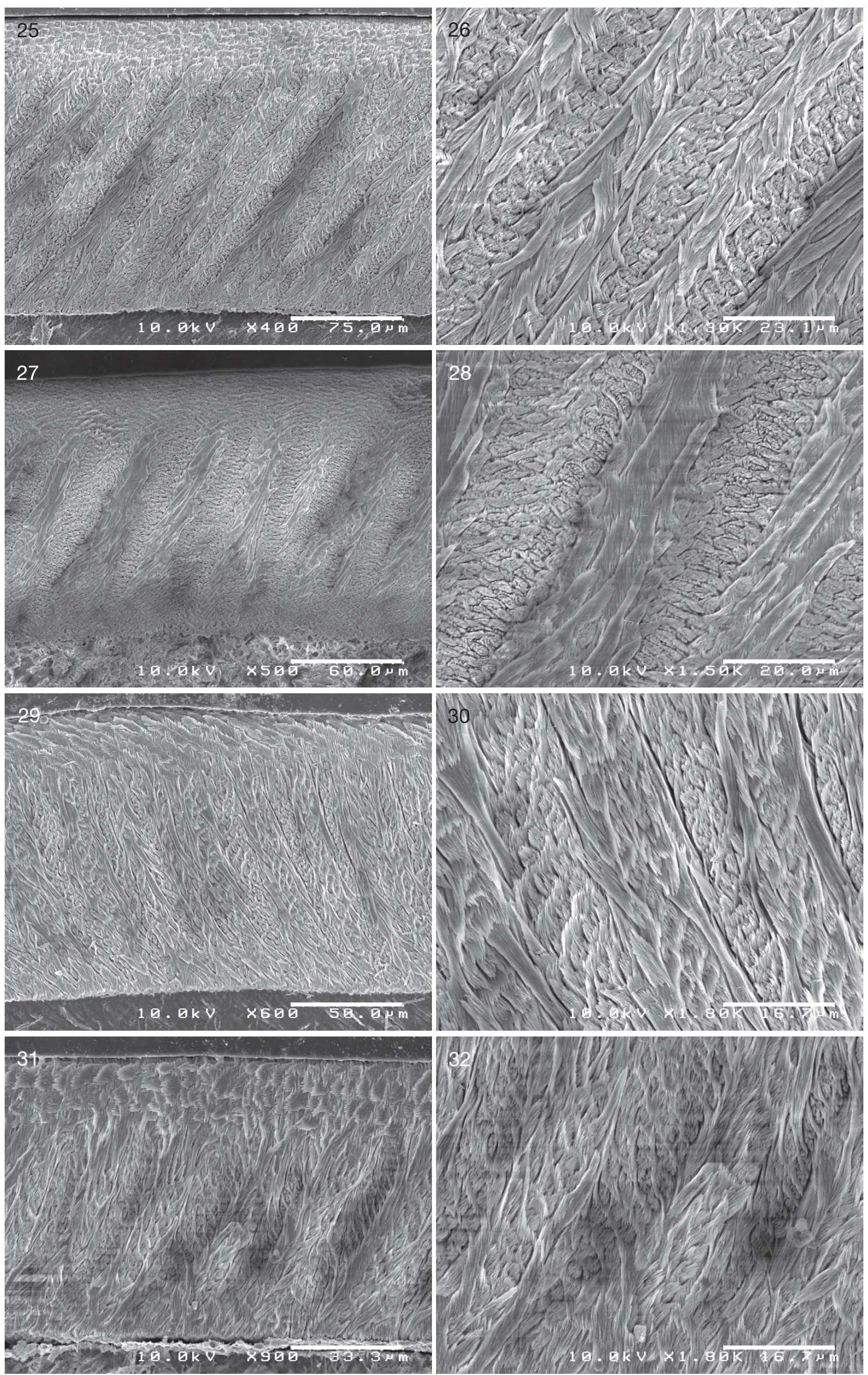


ANNEXE 1. - Suite.
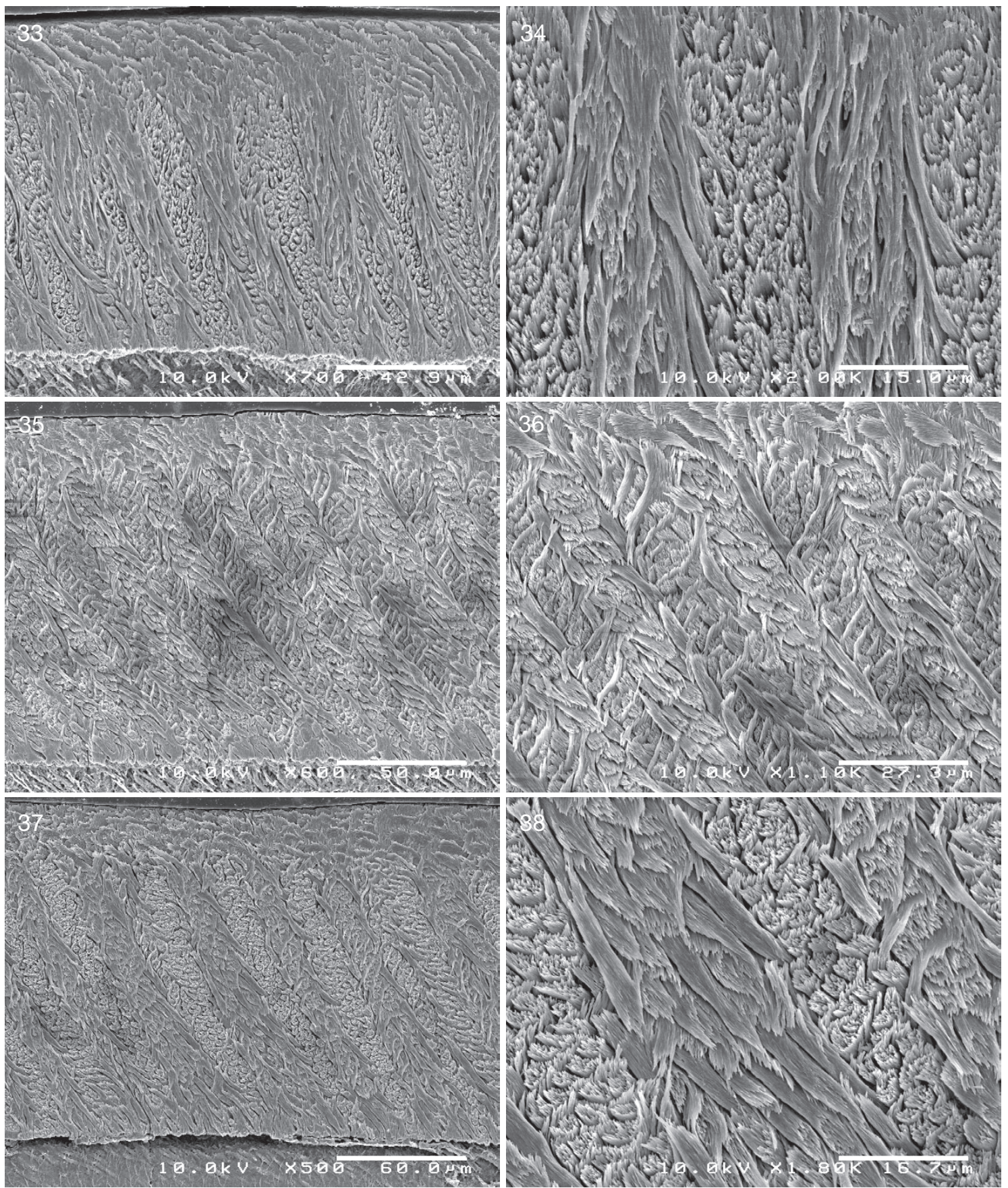

ANNEXE 1. - Images de microscopie à balayage de la structure de l'émail multisérié en coupe longitudinale chez les rongeurs de la région de Juanjui. 1, 2, MUSM 3519 (TAR-45): sous-type 1; 3, 4, MUSM 3526 (TAR-47): sous-type 2-3; 5, 6, MUSM 3527 (TAR-47): sous-type 2; 7, 8, MUSM 3528 (TAR-47): sous-type 1-2; 9, 10, MUSM 3529 (TAR-47): sous-type 2; 11, 12, MUSM 3530 (TAR-47): sous-type 2; 13, 14, MUSM 3531 (TAR-47): sous-type 2; 15, 16, MUSM 3533 (TAR49): sous-type 2-3; 17, 18, MUSM 3534 (TAR-49): sous-type 2-3; 19, 20, MUSM 3539 (TAR-50): sous-type 2-3; 21, 22, MUSM 3511 (TAR-55): sous-type 1-2; 23, 24, MUSM 3512 (TAR-55): sous-type 2; 25, 26, MUSM 3513 (TAR-55): sous-type 2; 27, 28, MUSM 3516 (TAR-55bis) : sous-type 2 ; 29, 30, MUSM 3517 (TAR55bis): sous-type 1-2; 31, 32, MUSM 3518 (TAR-55bis): sous-type 2; 33, 34, MUSM 3508 (TAR-56): sous-type 1; 35, 36, MUSM 3509 (TAR-56): sous-type 1; 37, 38, MUSM 3510 (TAR-56): sous-type 2. Les valeurs des échelles sont indiquées en bas à droite de chaque élément. 